EUROPEAN LABORATORY FOR PARTICLE PHYSICS

CERN-EP/98-163

2 October 1998

\title{
BEAM TESTS OF THE GAS ELECTRON MULTIPLIER
}

\author{
A. Bressan, J. C. Labbé, P. Pagano, L. Ropelewski, F. Sauli \\ (CERN, Geneva, Switzerland)
}

\begin{abstract}
We describe the results of systematic measurements, carried out with single and double GEM detectors with printed circuit read-out and having an active area of $10 \times 10 \mathrm{~cm}^{2}$, both in the laboratory and in a high energy charged particles beam at CERN. Using fast analogue readout electronics, we demonstrate efficiencies for minimum ionizing particles close to $100 \%$, with typical signal/noise ratios above 50 and up to $10^{3}$ for the single and double GEM configuration, respectively, and a time resolution of $15 \mathrm{~ns}$ fwhm. Localization accuracies around $40 \mu \mathrm{m}$ rms have been obtained for perpendicular tracks, degrading to $200 \mu \mathrm{m}$ at $20^{\circ}$ of incidence to the normal. Operated in a non-flammable gas mixture (argon-carbon dioxide), GEM detectors are robust, light and cheap to manufacture, and offer excellent performances and reliability suited for use in the harsh environments met at high luminosity colliders.
\end{abstract}

Submitted to

Nuclear Instruments and Methods in Physics Research

Corresponding author

fabio.sauli@cern.ch 



\section{INTRODUCTION}

The gas electron multiplier (GEM) consists of a thin polymer foil, metal-clad on both sides, and perforated by a high density of holes with a high-quality photo-lithographic processing. With a suitable difference of potential applied between the two sides, the composite mesh acts as an amplifier for electrons released by radiation in the overlying gas volume [1]. Originally capable only of moderate amplification factors, the device was used initially as a gain booster for a main amplifying structure (a micro-strip gas chamber, MSGC) [2]. Combined MSGC+GEM devices have been extensively tested in beam conditions [3,4] and have been adopted by the HERA-B experiment, in order to solve serious discharge problems encountered with the original design based on large size MSGCs [5]. Recent developments in the manufacturing technology, and a better understanding of the operating features, have led to the development of GEM electrodes offering proportional gains up and above $10^{4}$, therefore suitable for direct detection of small amounts of ionization on simple charge-collecting electrodes [3, 6, 7].

In a systematic research effort, motivated by the tracking requirements and the problems met at high luminosity colliders, we have built several GEM detectors with printed circuit board (PCB) read-out and tested their performances in the laboratory and with exposures to minimum ionizing particle beams at CERN. The high rate performances and the two-dimensional localization properties of GEM detectors are described separately $[8,9]$.

\section{CONSTRUCTION AND TEST OF THE DETECTORS}

One of the advantages of the GEM devices is that they are almost self-supporting, therefore permitting the realization of light detectors with minimum frame thickness. For the tests described here we have preferred however to use heavier but more flexible general purpose assemblies, based on gas-tight boxes holding the active elements and supporting the external readout and power distribution components (Fig. 1). The basic components of the assembly are two fiberglass frames with thin polymer windows, kept together with bolted screws; synthetic rubber joints guarantee the gas tightness. Feed-through printed circuits with the desired pattern permit the connection of the active pick-up elements to the external electronics; a coarser circuit is used for the HV contacts. The assembly boxes have signal feed-through on two sides, to permit bidimensional and small angle stereo readout (see Ref. [9]). A detector is then assembled inserting the readout board, wire-bonded to the feedthrough, and one or more GEM meshes; a drift electrode defines the sensitive volume and completes the device. GEM and drift electrodes are individually glued on thin frames, and supported on their corners with insulating pins, serving also as spacers to define the gaps. Miniature screws or soldered short wires ensure the electrical contact to the electrodes. Two inlets on opposite sides of the containment box permit to open-flux the detector with the operating gas. 
The picture in Fig. 2 gives a close view of the standard high-gain GEM geometry used for all measurements described here. Manufactured on $50 \mu \mathrm{m}$ thick kapton with $5 \mu \mathrm{m}$ copper on each side, the electrode has holes in parallel rows with $140 \mu \mathrm{m}$ between centers and a diameter of 70 $\mu \mathrm{m}$ at the metal sides and $55 \mu \mathrm{m}$ at the center of the kapton.

Figs. 3 and 4 show schematically the cross section of the single and double GEM detectors used for the measurements; both have an active area of $10 \times 10 \mathrm{~cm}^{2}$, with 512 pick-up strips at $200 \mu \mathrm{m}$ pitch. As shown in the figures, the operating voltages are applied to the GEM(s) through resistive dividers. Compared to a direct connection of each electrode to individual power supplies, this scheme is intrinsically safer since it guarantees, in case of current overloads, a fast drop in the difference of potential between the two GEM electrodes ${ }^{1}$. It has the inconvenience to fix the ratio between multiplying and transfer fields, and is therefore better suited for long-term operation than for systematic gain studies. In the single GEM, the upper gas layer constitutes the ionization and drift gap, and the lower layer the electron extraction and signal induction gap; in the double device, the region between the multipliers is referred to as transfer gap.

All GEM electrodes are HV tested before installation in the detector. The procedure consists in measuring the leakage current at slowly increasing voltages, applied through high value protection resistors; it is performed in a nitrogen box or in low-moisture air, inside a dust-free room. The standard acceptance criterion demands for less than $5 \mathrm{nA}$ leakage current at $500 \mathrm{~V}$ between the electrodes. This corresponds to an inter-electrode resistivity above $100 \mathrm{G} \Omega$, and has been found necessary and sufficient for a correct high-gain operation in the range of gas mixtures used. The few GEMs that do not pass the test are usually recovered after thorough cleaning or, in extreme cases, a second gentle metal etching.

\section{LABORATORY MEASUREMENTS}

The operating characteristics of the detector have been measured in the laboratory in a wide range of configurations, fields and gas fillings (see the quoted references); here we will report only selected results related to the beam run conditions. For the estimate of the proportional gain, and instead of a simple electronics calibration, subject to the well known dependence on the signal shape and amplifier's time constants (the socalled ballistic deficit), we have used an absolute method, consisting in the simultaneous measurement of current and rate under exposure to a known radiation source (soft $\mathrm{X}$-rays from a generator). Both in the single and double GEM structures, a fraction of the electron current is lost to the intermediate electrodes, depending on the field values [6, 10]; for a full gain characterization, the currents in all electrodes have therefore to be recorded. Figs. 5 and 6 show examples of "effective" gain plots for single and double GEM devices, deduced from a measurement of current on the pick-up electrode only; the real gain of each GEM at the field values used is about $20 \%$ larger. For the same reason, the combined gain in the double device is slightly smaller than the product of gains of the two elements. 
The gas mixture for these measurement was argon- $\mathrm{CO}_{2}$ in the volume proportions 70-30, the same used in the beam runs. In order to avoid discharges during calibration, in this measurements we did not push the voltages to their limits (for a detailed study of maximum gain and discharges under strong irradiation see Ref. [8]).

Some preliminary measurements of cluster size and efficiency were carried out in the laboratory using collimated X-ray and electron sources; although subject to various dispersions and losses not present in the beam runs, they cover a wider range of conditions and are therefore reported here. The dependence of the cluster size on field and geometry has been studied using collimated $5.9 \mathrm{keV}$ X-rays from an ${ }^{55} \mathrm{Fe}$ source. For all measurements, a single GEM device with PCB read-out was used, with pick-up strips $50 \mu \mathrm{m}$ wide at $200 \mu \mathrm{m}$ pitch (in later devices, namely those used in the beam runs, we have increased the strip width to $150 \mu \mathrm{m}$ ). The detector was operated in this case with an argon-DME 80-20 mixture, keeping both drift field and GEM voltage constant.

Fig. 7 gives an example of recorded total charge spectrum, and Fig. 8 shows the gain uniformity, measured across the detector. It can be seen that the local energy resolution, although not record breaking, is reasonably good $(20 \%$ fwhm). The gain uniformity is very good, about $\pm 10 \%$ over the active area; this is the expected consequence of the relative independence of gain from variations in the holes' size, observed for diameters below $80 \mu \mathrm{m}$, as discussed in Ref. [3].

The rate capability of GEM detectors has been analyzed in other works [6, 8]; Fig. 9 gives an example of the stability of absolute gain measured at increasing X-ray fluxes. No significant shifts are observed for irradiation rates up to $510^{5} \mathrm{~Hz} \mathrm{~mm}^{-2}$, demonstrating the absence of space charge or surface charging up phenomena in the chosen geometry and operating conditions.

The measured cluster size (at the base above threshold and fwhm) as a function of the induction field, and for several values of the induction gap is shown in Fig. 10. The small increase with the field can be explained by an increased spread of the avalanche at higher gains; the dependence from the gap is due to the combined effect of geometry (a wider induction profile) and of an increased diffusion. Keeping both induction and GEM voltages constant, we have measured the dependence of cluster size on the drift field (Fig. 11); the typical behavior of diffusion in argon-DME is found, with a minimum around $500 \mathrm{Vcm}^{-1}$, adding to the intrinsic dispersions due to the avalanche and to the induction process.

Exposing a single GEM detector, equipped with read-out electronics (see the next section) to a collimated ${ }^{90} \mathrm{Sn}$ beta source, we have measured the efficiency plateaux (Fig. 12). The fast electron component in the radiation was selected by a pair of scintillation counters in coincidence, placed downstream to the detector, to emulate minimum ionizing particles. The efficiency values indicated in the plot are relative, and subject to the well known fluctuations and limitations of this type of measurement; the plateau observed for the argon- $\mathrm{CO}_{2}$ mixture however overlaps quite well with the one measured in absolute terms during the beam exposure (see section 5.3). 
Although for the beam runs both the thickness of the induction gap and the value of the drift field were optimized for obtaining a minimum cluster size, the possibility of increasing this width adjusting geometry and field values is appealing in order to reduce the readout channels density, although at the expense of the two-track resolution. In view of the proposed possibility of using GEM as amplification element for an improved read-out of time projection chambers, an optimal adjustment of the cluster size to the desired pad response function is being thoroughly investigated experimentally and by calculation [11].

\section{TEST BEAM SET-UP}

Several GEM detectors partly equipped with fast read-out electronics were installed in the $\mathrm{X} 5$ beam at CERN, providing charged particle up to $250 \mathrm{GeV} / \mathrm{c}$ in momentum at a rate of around $10^{5}$ particles per second in two seconds spills. For the signals readout we have used groups of 64 adjacent channels on high-density amplifier cards based on the PreShape integrated chips [12], connected via twisted pair cables to a set of gated, charge integrating analogue-to-digital converters. The rise and decay times of the amplifiers are about 40 and $120 \mathrm{~ns}$, and we have integrated the signals with $200 \mathrm{~ns}$ wide gates to the ADCs. In all the beam measurements a gas mixture of argon and carbon dioxide in the volume percentages 70-30 was used. The existing beam facility includes a charged particle trigger for initiating the data acquisition, and a set of silicon micro-strip detectors, from which a full reconstruction of the expected hit position in the detector under test could be obtained. For each event, the detector raw ADC data as well as the full silicon telescope information were recorded for later analysis. During the available test beam period, several weeks in April '98, we have recorded around ten million triggers in various runs; around a half have been analyzed to study the effects of operating voltages and incidence angle, up to a maximum of 20 degrees to the normal. Only minimum monitoring was available during data taking, and most of the analysis was carried out off-line.

\section{EXPERIMENTAL RESULTS}

\subsection{Data structure and handling}

A typical raw event map of the charge recorded on 64 adjacent ADC channels, corresponding in space to $12.8 \mathrm{~mm}$, and for a track perpendicular to the single GEM detector is shown in Fig. 13; the fwhm of the cluster distribution is about 2 strips, $400 \mu \mathrm{m}$. The arrow indicates the predicted position of the track, deduced from the silicon micro-strip monitor. Fig. 14 gives an example of a more infrequent, wider cluster event, probably produced by a shower created by pions interacting upstream. Because of our use of a rather crude cluster-finding algorithm, such abnormal events, about $0.5 \%$ of the total, were rejected in the analysis by a cut on the cluster's width to $4 \sigma_{x}$ (rms of resolution), affecting correspondingly the estimate of efficiency. 
For calibration purposes, together with the beam data, two other samples were recorded: with a random trigger, in order to estimate the ADC pedestals, and with an external trigger sending fixed amplitude pulses to the amplifier cards, in order to calibrate their gain and the ADC slopes. The average value of the random trigger pulse height distribution is assumed to represent the pedestal for each channel, and is subtracted from the data; its gaussian root mean square represents the noise, $\sigma_{N}$, and corresponds, after taking into account the sensitivity of the amplifiers, to about 1200 electrons, with small variations between channels.

After pedestal subtraction, the program scans through the event and recognizes as a cluster any set of adjacent channels with content above $3 \sigma_{N}$; the weighted average over the cluster is assumed to represent the track position in strip units, and the number of adjacent channels above threshold the full cluster size. The sum of ADC counts within the cluster provides the overall detected charge for an event.

\subsection{Cluster size and total charge}

Figs. 15 and 16 give examples of the cluster size distributions, expressed in terms of strip number (the pitch is $200 \mu \mathrm{m}$ ) for the single and double GEM detectors, respectively, computed for typical runs at comparable values $(\sim 50)$ of the ratio signal/noise. The figures show both the conventionally defined cluster size (number of adjacent channels over threshold), and the fwhm of the distributions. Mostly as a consequence of the larger signal amplitude, and of the absence of the capacitively induced cathode signal pickup, the cluster size is wider than in micro-strip devices with similar geometry [13]. The evidence that there are no substantial dispersion mechanisms deriving from the GEM operation is obtained from the contour plots shown in Figs. 17 and 18, measured with the double GEM, and representing the total charge in the cluster (at the ADC input) as a function of the cluster size over threshold and of the fwhm of the cluster distribution. Vide clusters above threshold correspond simply to larger signals; the fwhm of the charge distribution appears instead to be almost independent from the total pulse height.

Figs. 19 and 20 show the distribution of the total charge, summed over the cluster, for minimum ionizing particles perpendicular to the detector's plane in the single and double GEM; the small peaks on the left represent the electronics noise. The measurements have been carried out in the middle of the operating voltage range (at a signal/noise ratio of 50 and 150 , respectively).

\subsection{Detection efficiency and localization accuracy}

Making use of the predicted track position provided by the silicon micro-strip telescope, we have computed the absolute efficiency and the position resolution as a function of voltage for the single GEM detector (Figs. 21 and 22), and for the double GEM (Figs. 23 and 24). In the latter case, the two multipliers were operated at equal difference of potentials, summed in the abscissa. The acceptance criterion for an event is finding a 
track within 4 standard deviations in the difference of coordinates (about \pm $200 \mu \mathrm{m}$ ). Figs. 21 and 23 provide also values of the signal over noise, defined as ratio of most probable value of the total detected charge to the single channel noise. One can see that for the single GEM the $98 \%$ efficiency level is reached at $530 \mathrm{~V}$ (signal/noise of 20); the plateau extends up to $570 \mathrm{~V}$. By comparison with Figs. 5, one can infer that full efficiency is reached in our operating conditions at an effective gain above 2000. In the double GEM, the efficiency plateau begins at similar values of gain (reached with $410 \mathrm{~V}$ on each GEM, see Fig. 6), but extends to considerably larger values, up to a signal/noise ratio exceeding $10^{3}$.

The single-track localization accuracy, that includes the nonsubtracted contribution of the silicon telescope, is $\sim 45 \mu \mathrm{m} \mathrm{rms} \mathrm{for} \mathrm{the}$ single GEM in the middle of the efficiency plateau, and $\sim 40 \mu \mathrm{m}$ for the double (Figs. 22 and 24). The small improvement is probably a consequence of the increased pulse height; this result demonstrates the absence of additional dispersive effects due to the use of a multiple device. The values compare well to the best localization accuracy obtained in similar conditions with MSGCs [14]. The cluster size, on the other hand, steps from $\sim 380$ to $\sim 420 \mu \mathrm{m}$ fwhm between single and double GEM, at similar values of gain; this is consistent with the increased diffusion in the longer drift path (the $2 \mathrm{~mm}$ additional transfer gap). Note also the agreement between the beam results and the laboratory measurements for $1 \mathrm{~mm}$ induction gaps, Fig. 10, notwithstanding the different gas composition. From the observations, and the knowledge of the diffusion properties in the gas used, one can deduce that the width of the signal induction is determined by geometry (thickness of the gap) and electron diffusion in the gas; avalanche dispersions in the multiplier are negligible.

Given the relatively wide cluster size, we have emulated the response of a detector with wider strips adding by software pairs of adjacent channels before the event reconstruction. The resolution is not affected by the merging, as shown by Figs. 25 and 26 that provide accuracy distributions for the original $(200 \mu \mathrm{m})$ and grouped $(400 \mu \mathrm{m})$ double GEM data; the spectra have comparable rms $(\sim 40 \mu \mathrm{m})$. At the expense of a reduced two-track resolution, a cost-effective decrease by a factor of two in the number of read-out channels is therefore possible without degradation of the localization accuracy of the detector.

We have gone to the extreme in the analysis emulating the effect on resolution of a digital read-out, each channel being considered on or off depending on its content in comparison with a pre-set threshold. The track coordinate is then estimated as the middle point for each digital cluster. The resulting distribution of position accuracy is shown in Fig. 27; it has a standard deviation of $\sim 60 \mu \mathrm{m}$, well matching the one expected for a 200 $\mu \mathrm{m}$ pitch (the classic square root of 12 factor). The result is almost independent of the threshold value adopted, from 3 to 7 times the rms of the noise; the efficiency varies from $99.6 \%$ to $98.7 \%$. A digital read-out has been considered by several groups for MSGC systems in order to substantially reduce electronics costs, but turned out to be rather critically dependent on the threshold values for the moderate gains that could be achieved. This appears not to be the case for the large gain GEM devices. 
The angular response of efficiency and resolution has been studied rotating in small increments the chambers around a vertical axis, parallel to the readout strips; no effort was done to mechanically align the two detectors, and the difference distributions to the predicted track were centered by software. Due to mechanical constraints, only a maximum angle of about $20^{\circ}$ to the normal could be reached. Figs. 28 and 29 show, as a function of the rotation angle, the position accuracy (rms) and the most probable value of the total charge for minimum ionizing tracks, for the single and double GEM detectors operated in the middle of their efficiency plateau. The degradation of accuracy with the incidence angle is similar to the one observed with MSGCs [15], and is a natural consequence of the dispersions due to the primary ionization statistics. As expected, the most probable value of the detected charge varies only very little over the angular range covered. Operating the detectors in a DME-rich mixture (as against argon- $\mathrm{CO}_{2}$ ) would improve the accuracy at large angles; for technical reasons however this was not possible in the runs.

\subsection{Time resolution}

To study the intrinsic time resolution of the double GEM detector for fast particles, in some runs we have used analogue fan-outs to split the signals at the PreShape output, and measured simultaneously the charge and, after discrimination, the time for each channel (referred to the trigger) with a set of TDCs. The discrimination threshold used, $30 \mathrm{mV}$, corresponds to around $50 \mathrm{nC}$ at the ADC signal input (or $10 \sigma_{\mathrm{N}}$, see Fig. 20). For each event, we have then computed the total cluster charge and the shortest recorded time within the strips in the cluster. The scatter plot in Fig. 30 shows the correlation between the time information (in ns) and the total charge; it has the characteristic shape determined by electronics slewing. The projection of the distribution on the time axis, Fig. 31, has a fwhm of about $24 \mathrm{~ns}$. The time resolution can be improved with a software correction to the slewing, made making use of the measured correlation (Fig. 32); the resulting projection (Fig. 33) has a fwhm of $15 \mathrm{~ns}$, and corresponds to the intrinsic time resolution of the detector. This is at least a factor of two better than the typical results obtained with MSGCs, owing to their rather poor signal to noise characteristics [16]. Note that these results, essentially dominated by the energy loss statistics, have been obtained in conditions of a relatively small drift velocity, and can certainly be improved with a better choice of gas and drift fields.

\section{CONCLUSIONS AND SUMMARY}

In systematic laboratory tests and exposures to minimum ionizing particles beam, we have studied the response of detectors using as gaseous amplification element one or two GEM meshes in cascade. Fast ( $\sim 40 \mathrm{~ns}$ shaping time) amplifiers have been used for all this work to cope with the LHC requirements; for most of the measurements a safe, non-flammable mixture of argon and carbon dioxide was used. Signals are detected collecting the electron charge, drifting out of the GEM amplification 
channels, on a thin printed circuit boards with strips $200 \mu \mathrm{m}$ apart. Typical signal/noise ratios up to 70 are reached in the single GEM device and up to $10^{3}$ for the double GEM, with efficiencies close to $100 \%$. Localization accuracies around $40 \mu \mathrm{m} \mathrm{rms}$ are obtained for tracks perpendicular to the detector, degrading to $\sim 200 \mu \mathrm{m}$ for tracks at $20^{\circ}$ to the normal. Both the single and the double GEM detectors seem to satisfy the requirements of efficient operation in high intensity minimum ionizing particles beam. The considerably wider operating margins of the double GEM, however, suggest an intrinsically safer operation in harsh conditions, in presence of high fluxes and of a background of heavily ionizing particles, as studied extensively and described in a separate paper [8].

\section{ACKNOWLEDGMENTS}

L. Schmitt, I. Konorov, S. Masciocchi, M. Beck and S. Gerassimov, in the COMPASS collaboration, have actively contributed to the setting-up of the data acquisition and to the ensuing analysis for the beam runs. The GEM manufacturing technology has been developed by A. Gandi, L. Mastrostefano and R. De Oliveira (CERN-EST-MT). The wire bonding for the inter-connections of the detectors to the read-out electronics have been made by O. Runolffson and Muhlemann (CERN-EP-OPAL). The essential contribution of all to the realization of the present work is here warmly acknowledged.

\section{REFERENCES}

[1] F. Sauli, Nucl. Instrum. Methods A386 (1997) 531.

[2] R. Bouclier, W. Dominik, M. Hoch, J.C. Labbé, G. Million, L. Ropelewski, F. Sauli, A. Sharma, G. Manzin, Nucl. Instrum. Methods A396 (1997) 50-66.

[3] J. Benlloch, A. Bressan, C. Büttner, M. Capeáns, M. Gruwé, M. Hoch, J.C. Labbé, A. Placci, L. Ropelewski, F. Sauli, A. Sharma, R. Veenhof, IEEE Trans. Nucl. Sci. NS-45 (1998) 234.

[4] W. Beaumont, T. Beckers, J. DeTroy, V. Van Dyck, O. Bouhali, F. Udo, C. VanderVelde, W. Van Doninck, P. Vanlaer, V. Zhukov, Studies of an MSGC equipped with a GEM grid as a tracking device, Wire Chamber Conference (Vienna, Feb. 23-27, 1998). Subm. Nucl. Instrum. Methods (1998).

[5] B. Schmidt, Microstrip Gas Chambers: recent developments, radiation damage and long term behaviour, Wire Chamber Conference (Vienna, Feb. 23-27, 1998). Subm. Nucl. Instrum. Methods (1998).

[6] J. Benlloch, A. Bressan, M. Capeáns, M. Gruwé, M. Hoch, J.C. Labbé, A. Placci, L. Ropelewski, F. Sauli, Further developments of the Gas Electron Multiplier (GEM), Wire Chamber Conference (Vienna, Feb. 22-26, 1998). CERN-EP/98-50. Subm. Nucl. Instrum. Methods (1998).

[7] C. Büttner, M. Capeáns, W. Dominik, M. Hoch, J.C. Labbé, G. Manzin, G. Million, L. Ropelewski, F. Sauli, A. Sharma, Nucl. Instr. Methods A409 (1998) 79. 
[8] A. Bressan, M. Hoch, P. Pagano, L. Ropelewski, F. Sauli, S. Biagi, A. Buzulutskov, M. Gruwé, A. Sharma, D. Moermann, G. De Lentdecker, High rate behavior and discharge limits in micro-pattern detectors, CERNEP/98-139. Subm. Nucl. Instrum. Methods (1998).

[9] A. Bressan, L. Ropelewski, F. Sauli, D. Mörmann, T. Müller, H.J. Simonis, Two-dimensional readout in GEM detectors, Subm. Nucl. Instrum. Methods (1998).

[10] R. Bellazzini, A. Brez, G. Gariano, L. Latronico, N. Lumb, G. Spandre, M.M. Massai, R. Raffo, M.A. Spezziga, What is the real gas gain of a standard GEM?, Wire Chamber Conference (Vienna, Feb. 23-26, 1998). INFN PI/AE 98/01. Subm. Nucl. Instrum. Methods (1998).

[11] M. Gruwé, W. Klempt, F. Sauli, A. Sharma, Measurements on a small TPC prototype with GEM read-out, in preparation (1998).

[12] J.F. Clergeau, D. Contardo, R. Haroutunian, G. Smadja, LYCEN/9424 (1994).

[13] R. Bouclier, M. Capeáns, C. Garabatos, G. Manzin, A. Peisert, L. Ropelewski, F. Sauli, J.C. Santiard, L.I. Shekhtman, T. Temmel, G. Fischer, Proc. Int. Conf. on Micro-Strip Gas Chambers (Legnaro, October 13-15, 1994) 79.

[14] A. Barr, et al., Nucl. Instrum. Methods A403 (1998) 31-56.

[15] F.D. van den Berg, F.G. Hartjes, J. Schmitz, F. Udo, A.R.d. Winter, B. Dutrieue, C.V. Velde, Nucl. Instrum. Methods A349 (1994) 438.

[16] J.F. Clergeau, D. Contardo, T. Ladzinsky, Proc. Int. Workshop on Micro-Strip Gas Chambers (Legnaro, October 13-14, 1994) 64. 


\section{FIGURE CAPTIONS}

Fig. 1: General view of a GEM detector. The active electrodes (GEMs, drift plane, readout boards) are separately mounted on thin frames, and assembled within a general purpose gas containment vessel with signal feed through. The active area is $10 \times 10 \mathrm{~cm}^{2}$.

Fig. 2: Close view of a GEM electrode. Circular holes $70 \mu \mathrm{m}$ in diameter are chemically etched in offset rows at a pitch of $140 \mu \mathrm{m}$.

Fig. 3: Schematics of a single GEM detector with a stripped printed circuit board read-out. The pitch is $200 \mu \mathrm{m}$.

Fig. 4: Schematics of the double GEM detector and of the corresponding HV distribution networks.

Fig. 5: Effective gain curve of a single GEM, operated in argon- $\mathrm{CO}_{2}$ 70-30, for typical values of drift and induction fields.

Fig. 6: Double GEM detector: effective gain of each electrode and of the combined device, operated with the two multipliers kept at equal potential differences.

Fig. 7: Pulse height distribution for $5.9 \mathrm{keV}$ obtained with a single GEM detector at a gain of 5000 .

Fig. 8: Gain uniformity across the single GEM detector.

Fig. 9: Gain as a function of rate in the double GEM detector under soft Xray irradiation.

Fig. 10: Cluster size (above threshold and fwhm) in the single GEM, measured with $5.9 \mathrm{keV}$-rays as a function of field and for several thickness of the induction gap.

Fig. 11: Dependence of the cluster size on the drift field for a single GEM detector, operated at constant gain and induction field.

Fig. 12: Relative efficiency curves measured for the single GEM detector in various gas mixtures, using a collimated ${ }^{90} \mathrm{Sn}$ electron source.

Fig. 13: Typical charge profile for a beam track, recorded with the single GEM detector. The arrow shows the predicted position of the track.

Fig. 14: A rare event with a wide cluster, probably due to a hadronic shower generated upstream from the detector.

Fig. 15: Cluster size distribution (over threshold and fwhm) for minimum ionizing tracks, perpendicular to the single GEM detector.

Fig. 16: Cluster size distribution (over threshold and fwhm) for minimum ionizing tracks, perpendicular to the double GEM detector.

Fig. 17: Correlation plot of the cluster size over threshold and total charge at the ADC input.

Fig. 18: Correlation plot of cluster size (fwhm) and total charge.

Fig. 19: Total cluster charge for minimum ionizing tracks, and noise spectrum recorded with the single GEM detector.

Fig. 20: Total cluster charge noise spectrum for the double GEM detector. Fig. 21: Detection efficiency for minimum ionizing particles and ratio signal over noise as a function of voltage in the single GEM detector. Fig. 22: Localization accuracy and cluster size (fwhm) as a function of voltage of the single GEM detector.

Fig. 23: Efficiency and signal over noise in the double GEM detector.

Fig. 24: Localization accuracy and cluster size (fwhm) in the double GEM. 
Fig. 25: Space accuracy distribution computed from the center of gravity of the original charge recorded on strips at $200 \mu \mathrm{m}$ pitch.

Fig. 26: Space accuracy distribution computed after adding the charge in adjacent pairs of strips (corresponding to $400 \mu \mathrm{m}$ pitch).

Fig. 27: Space accuracy obtained estimating the position from a digital count of strips over threshold.

Fig. 28: Space accuracy and most probable total charge as a function of the track's angle to the normal for the single GEM detector.

Fig. 29: Space accuracy and most probable total charge as a function of the track's angle to the normal for the double GEM detector.

Fig. 30: Correlation plot of the total charge and of the time at threshold. Fig. 31: Projection of the previous plot on the time axis (fwhm $23 \mathrm{~ns}$ ).

Fig. 32: Correlation plot of time and total charge, after correction for the correlation provided by the data in Fig. 28.

Fig. 33: Projection of the previous plot on the time axis; the distribution has a fwhm of $17 \mathrm{~ns}$. 



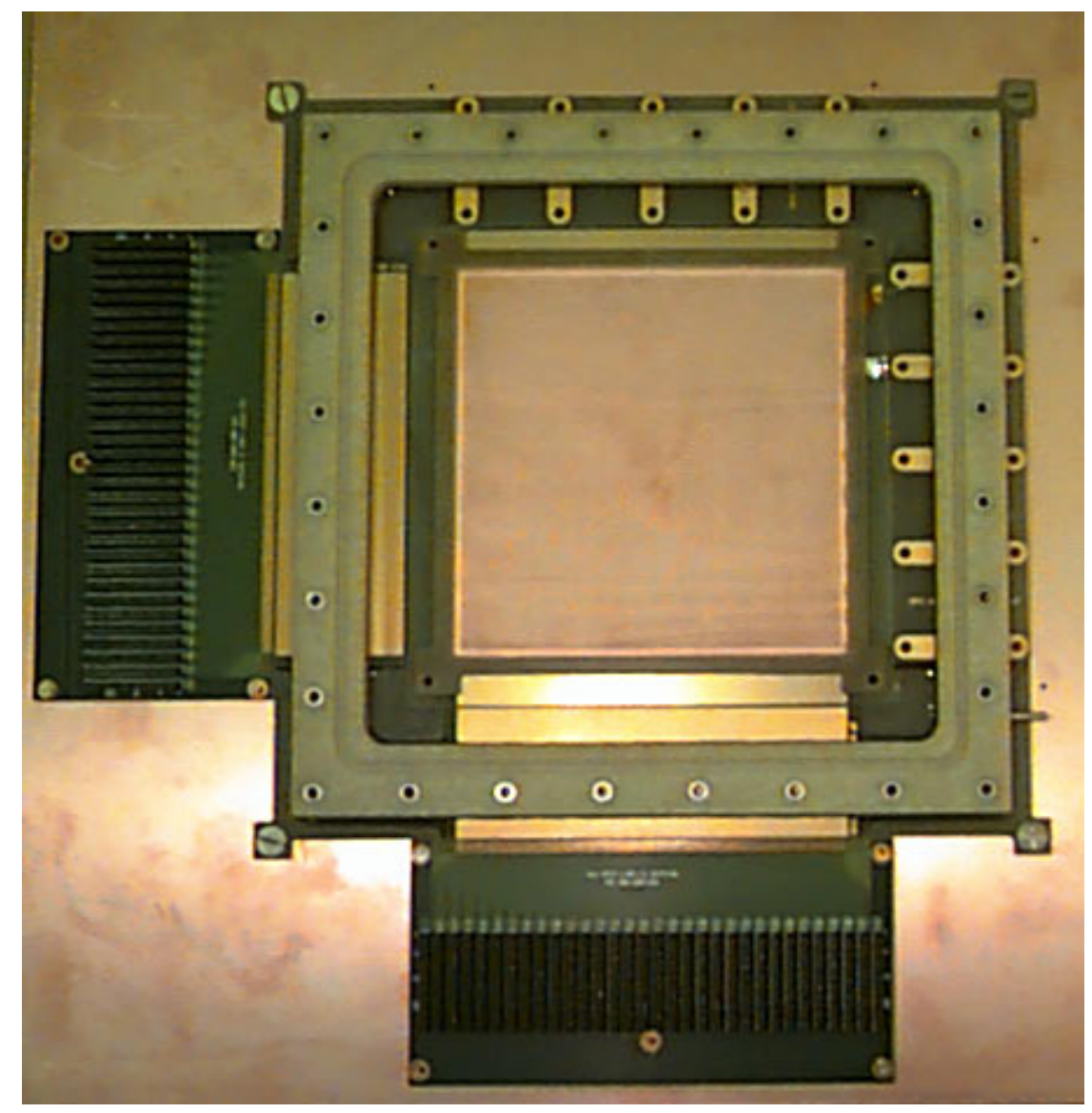

Fig. 1

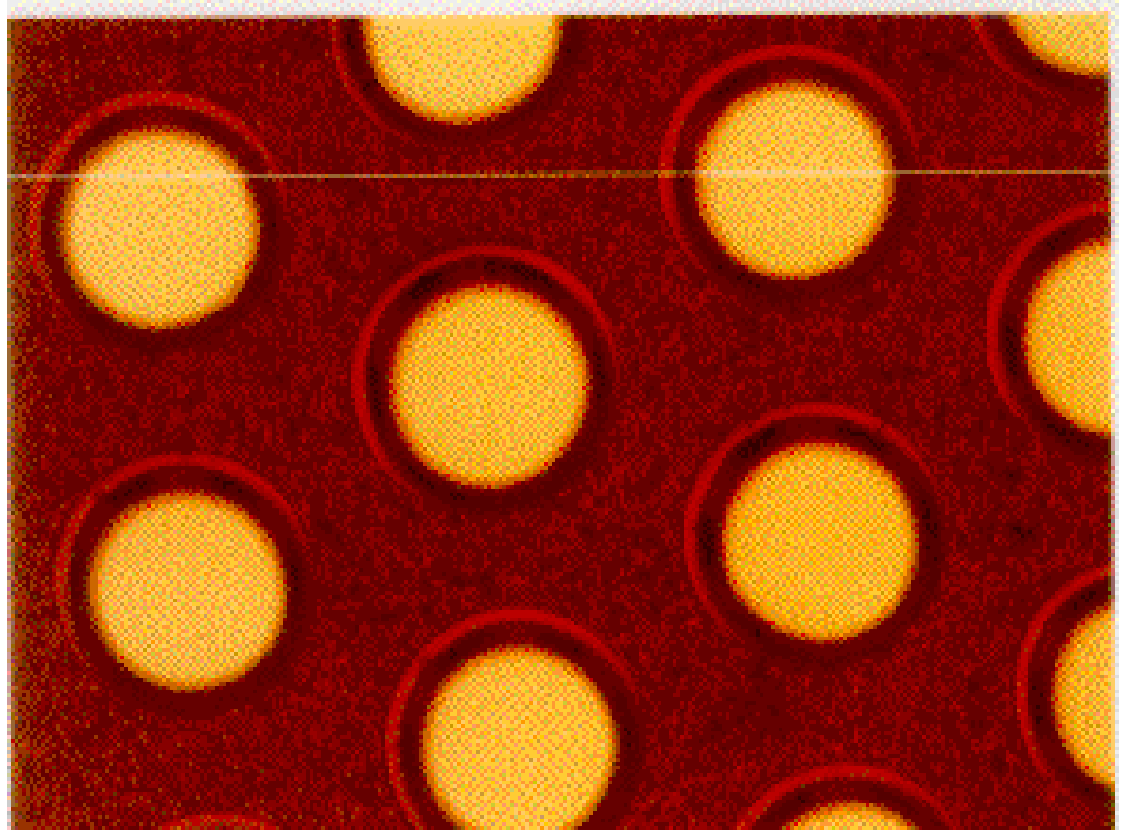

Fig. 2 


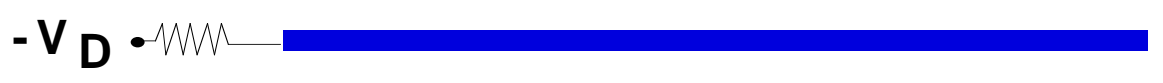

$3 \mathrm{~mm}$

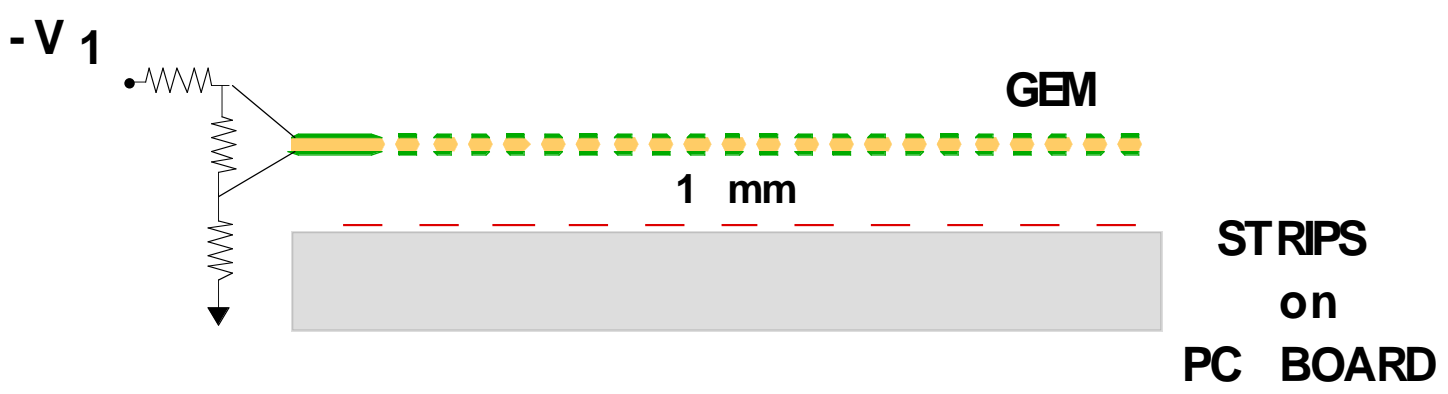

Fig. 3
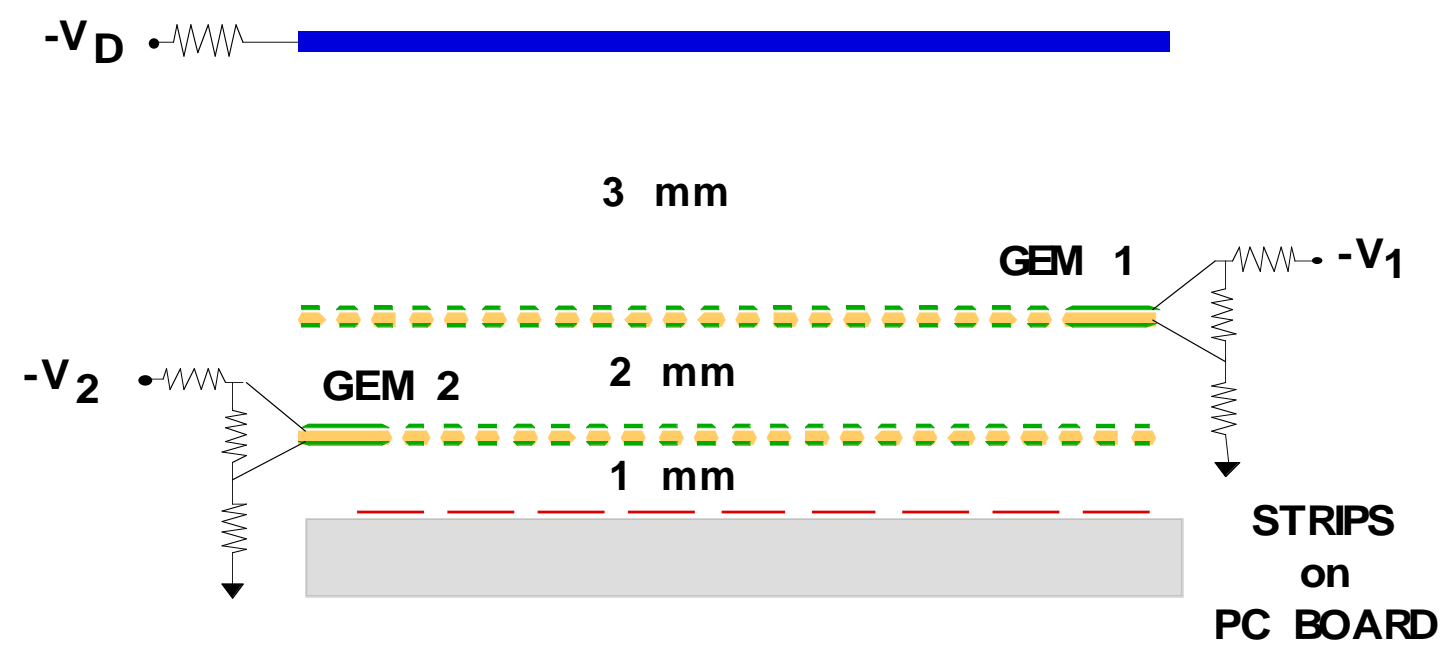

Fig. 4 


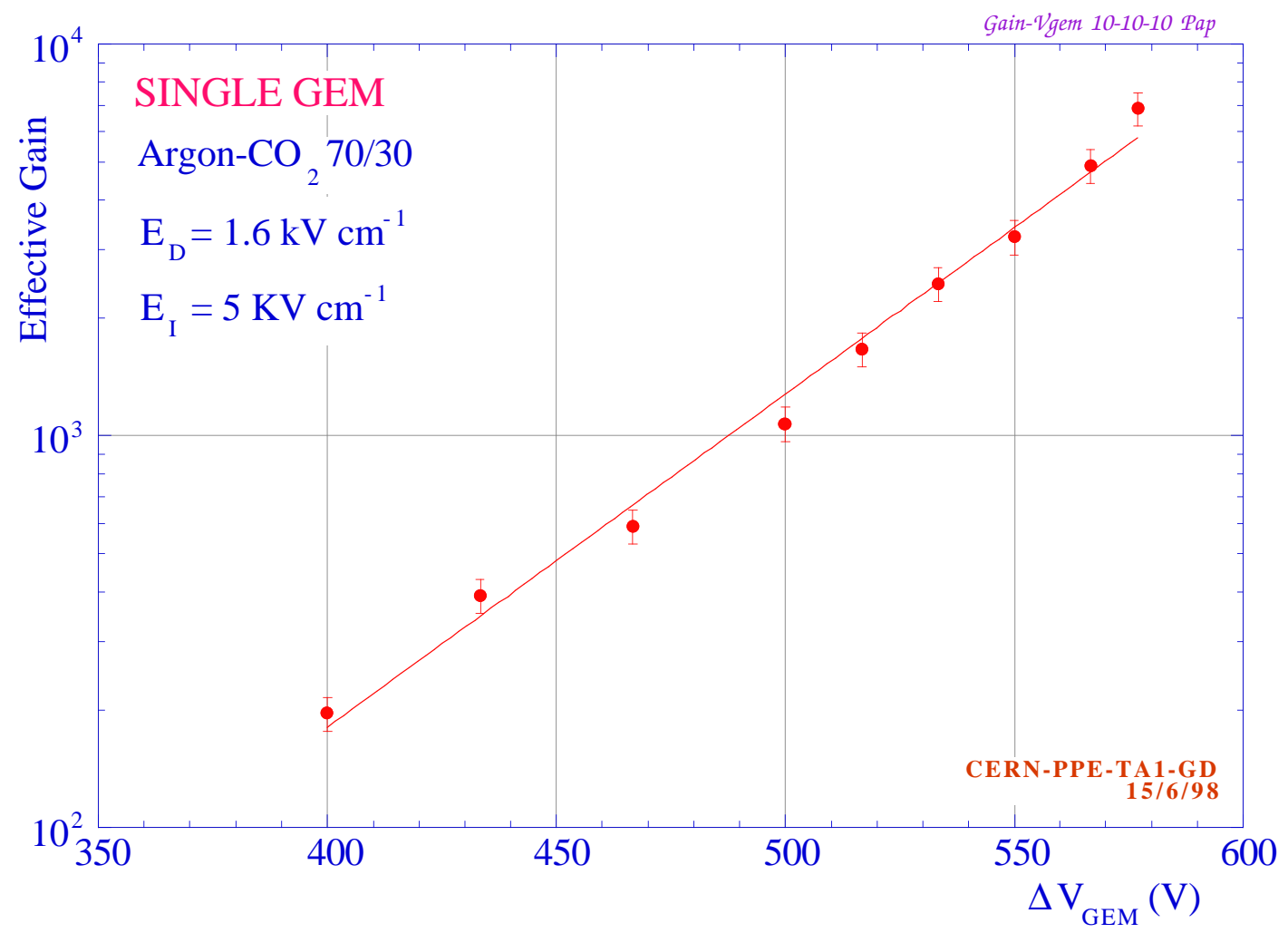

Fig. 5

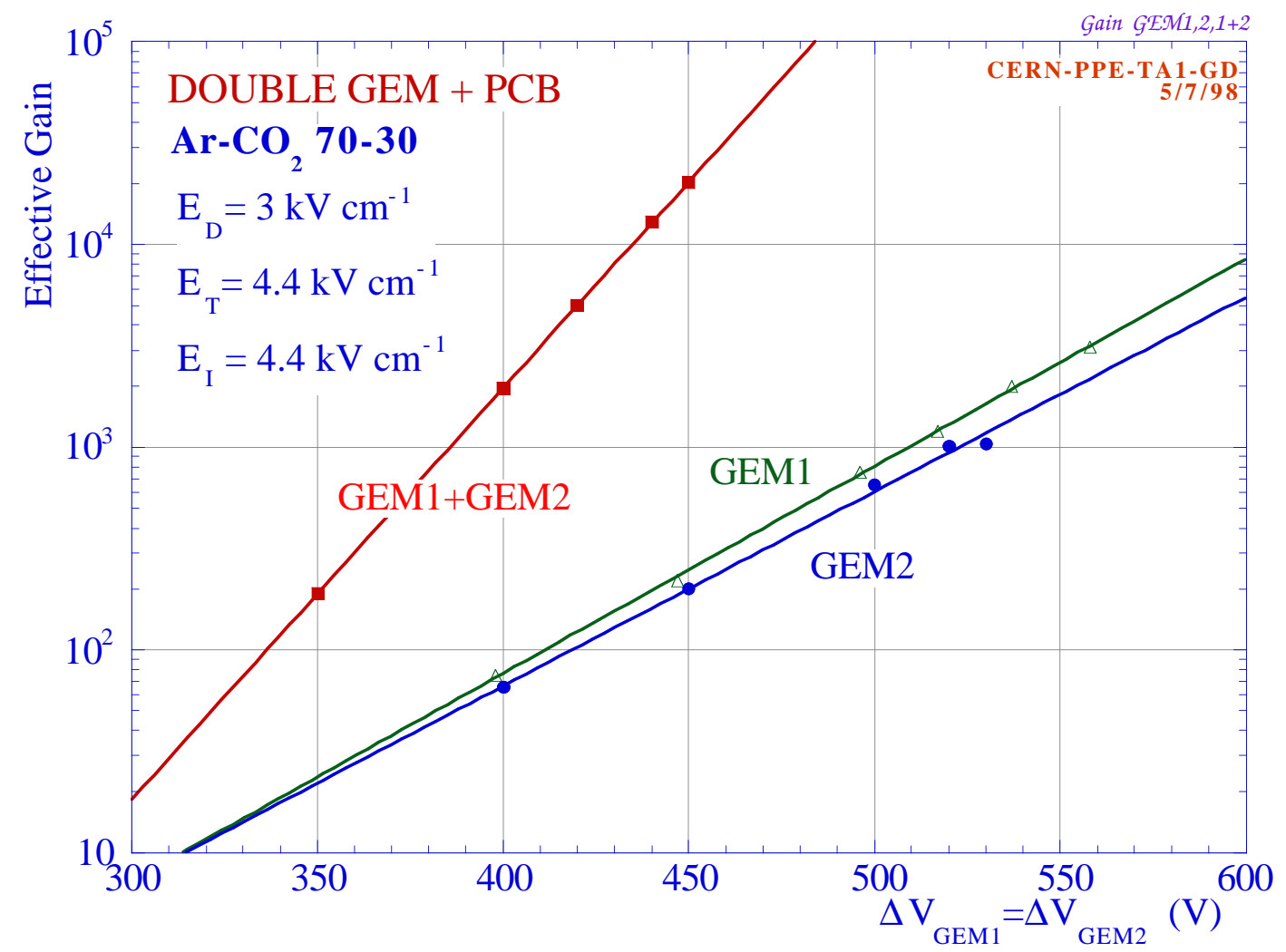

Fig. 6 


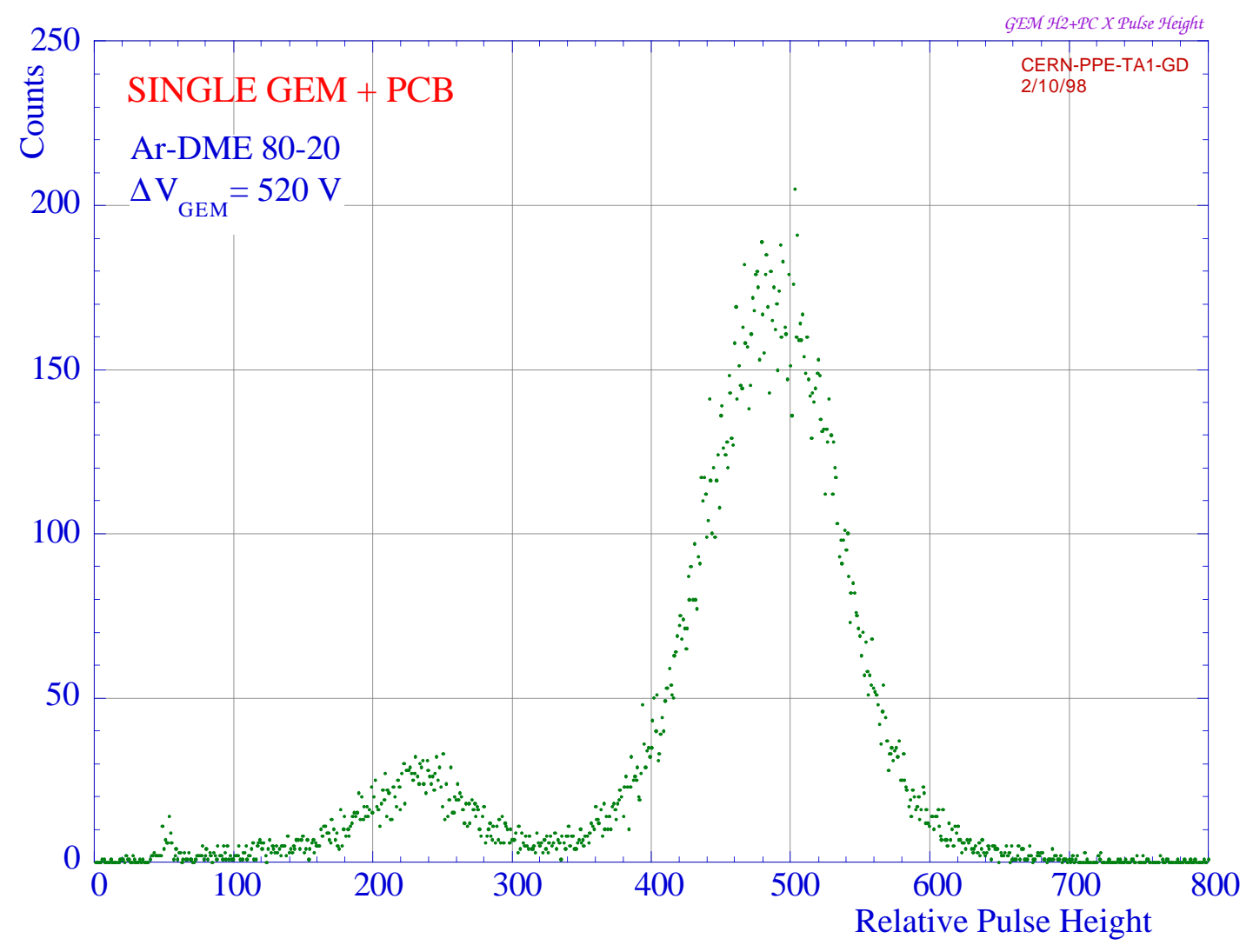

Fig. 7

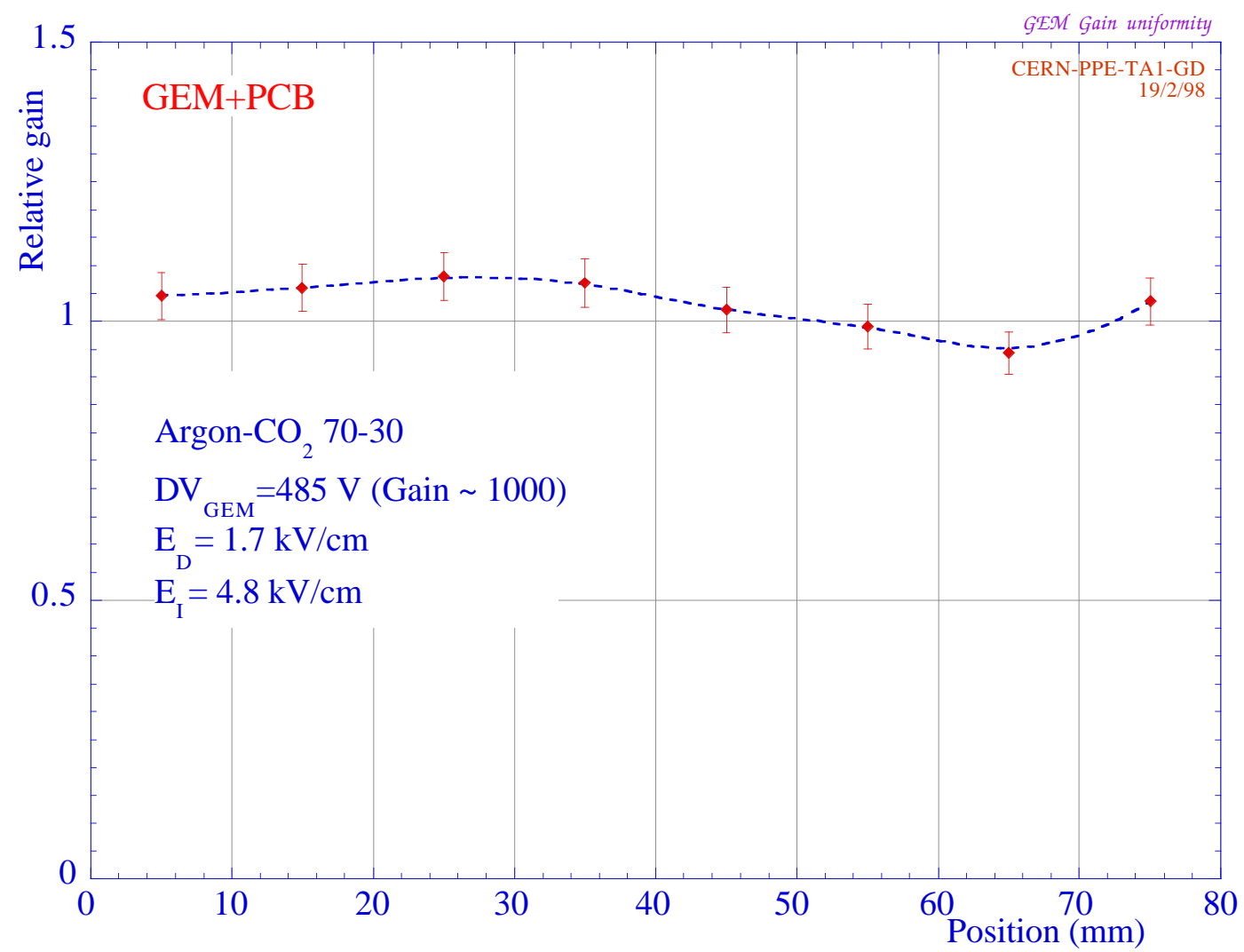

Fig. 8 
DGEM Gain-Rate

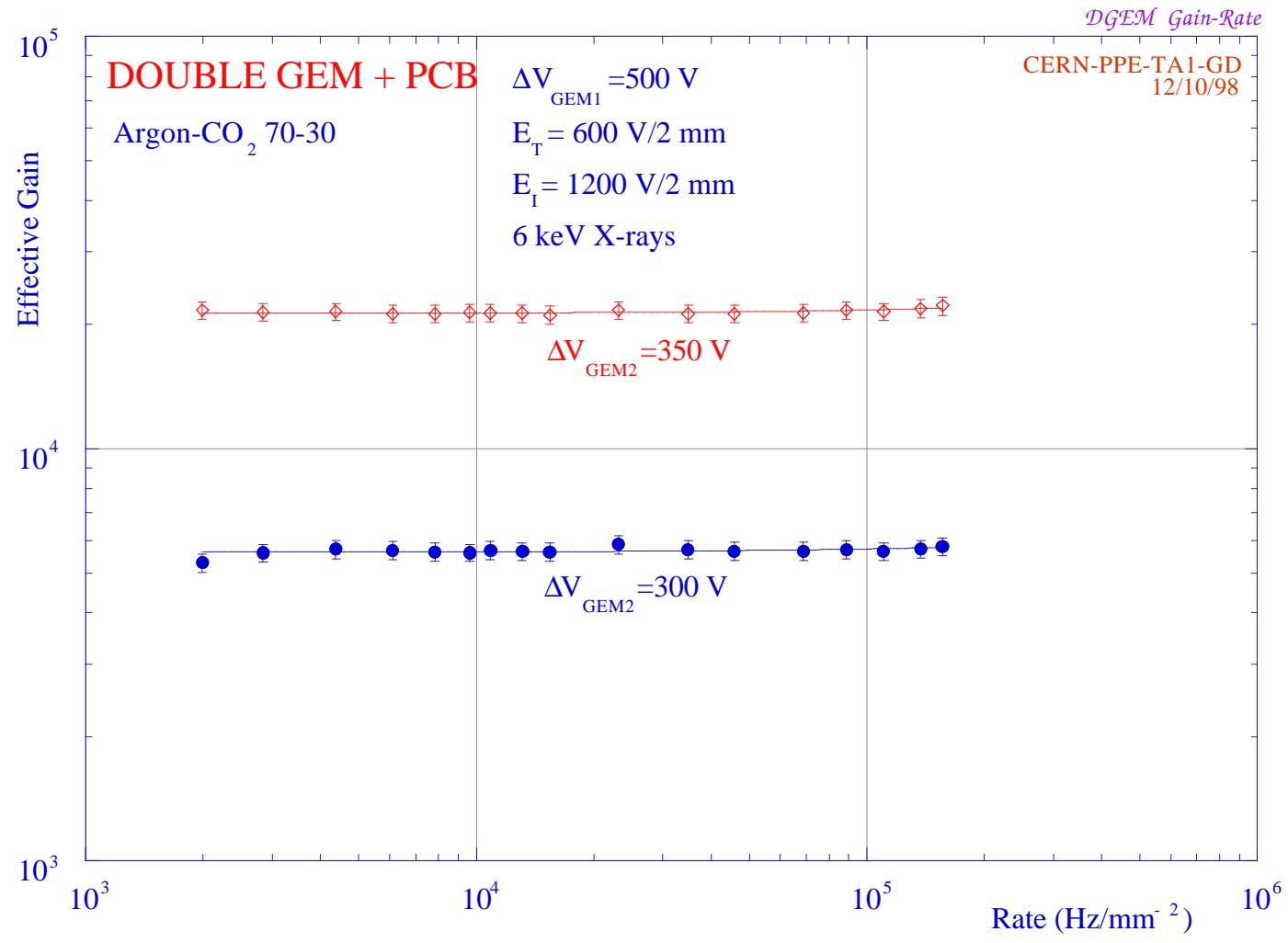

Fig. 9

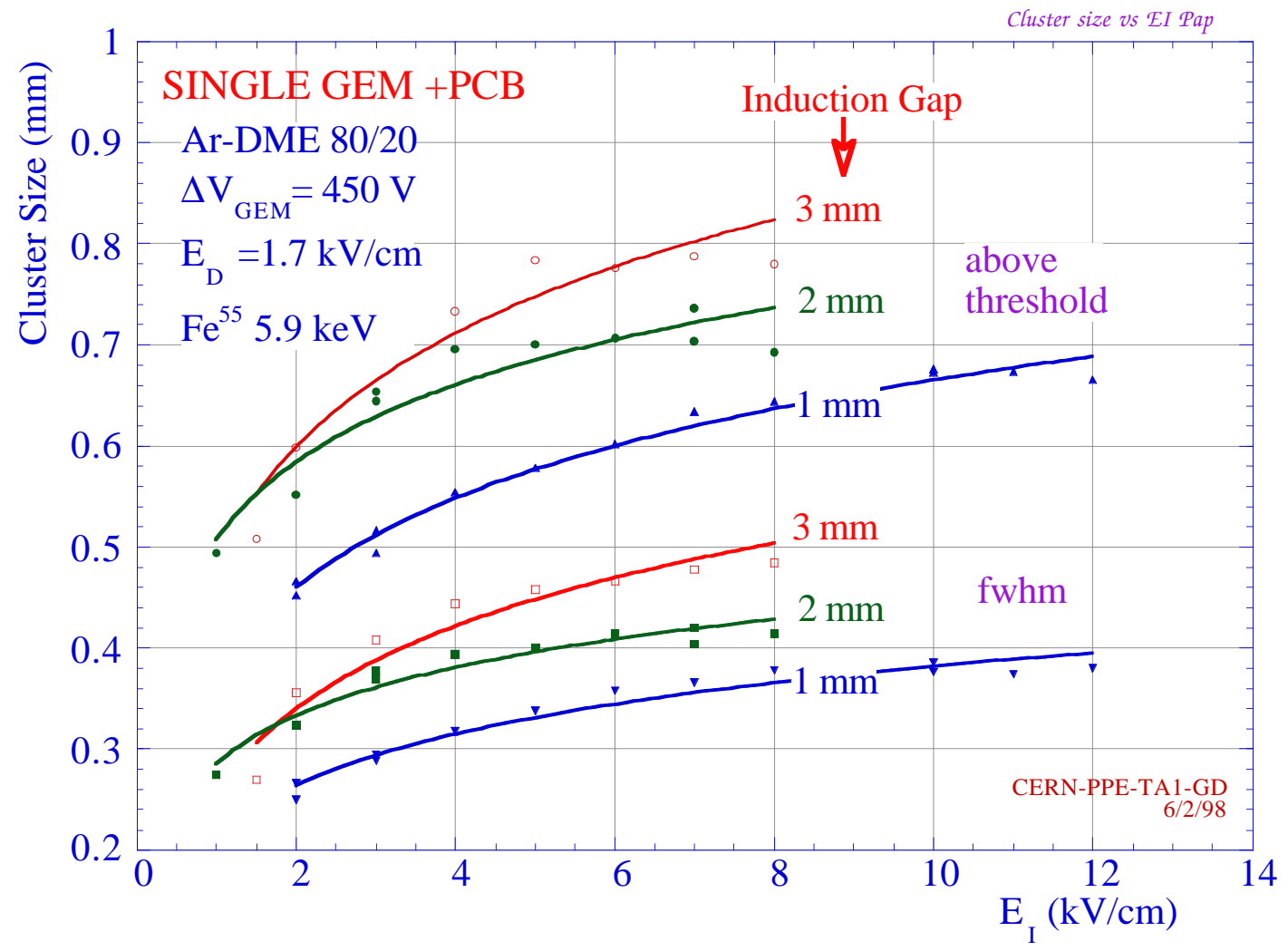


Fig. 10

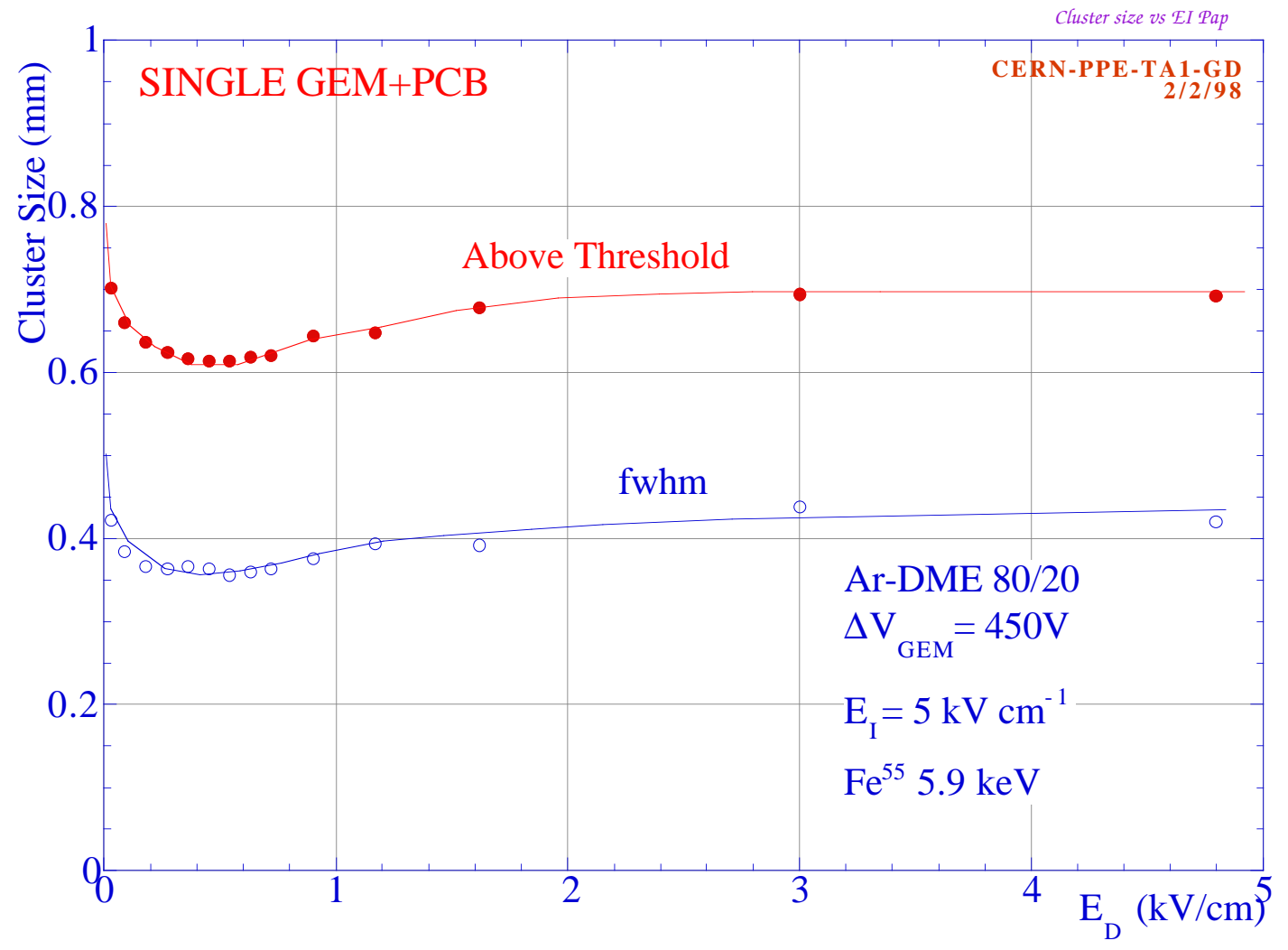

Fig. 11

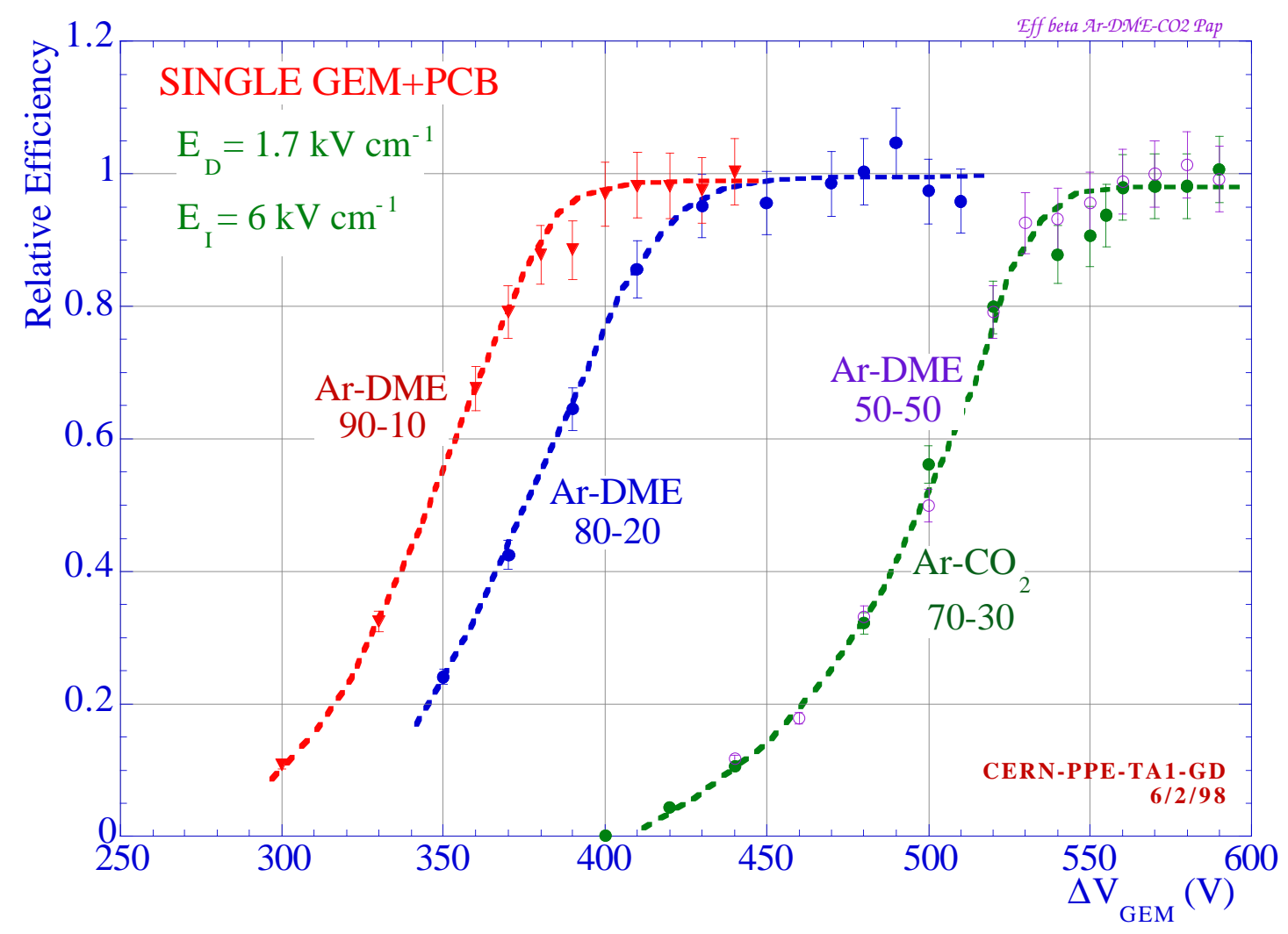


Fig. 12

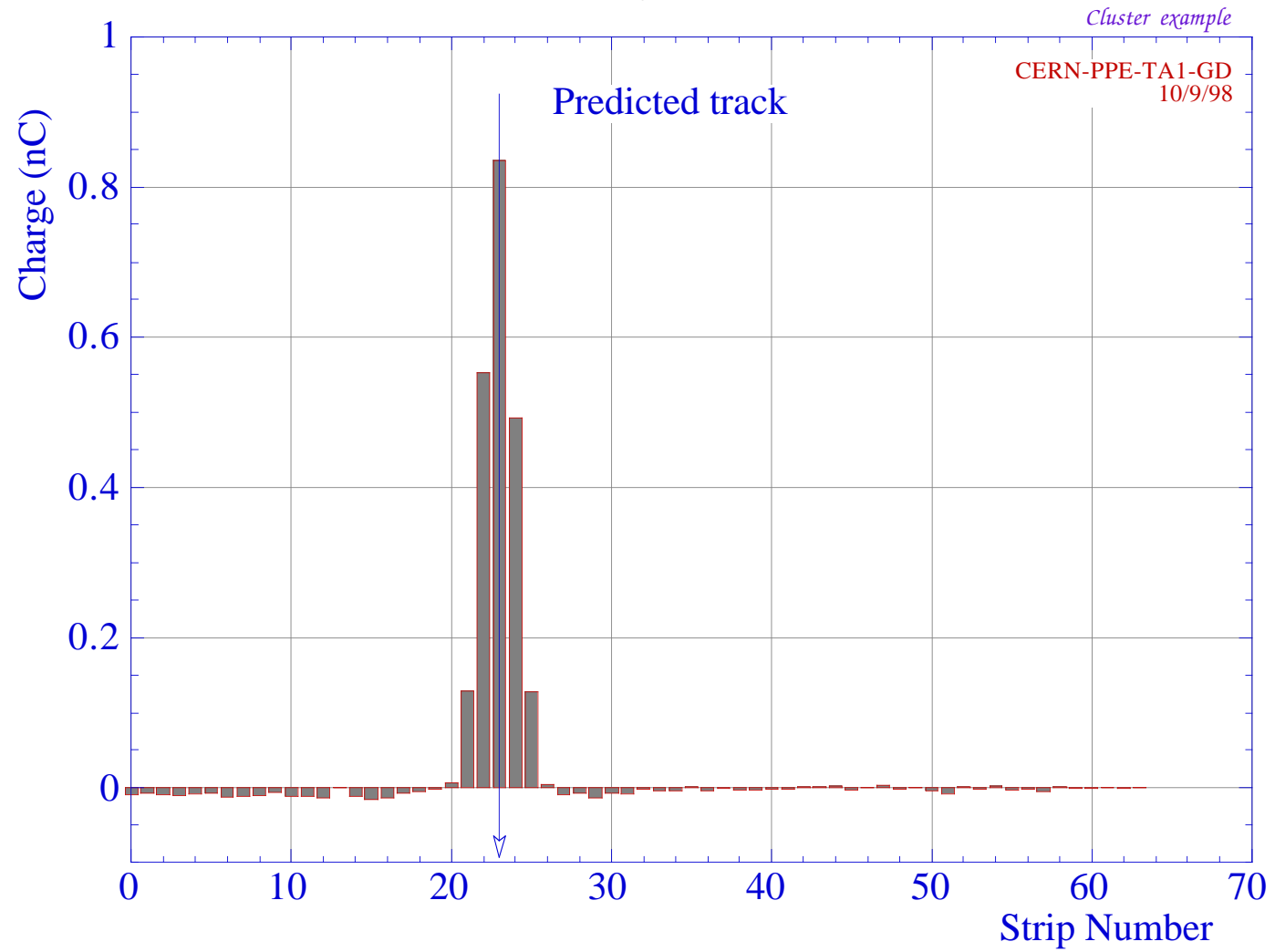

Fig. 13

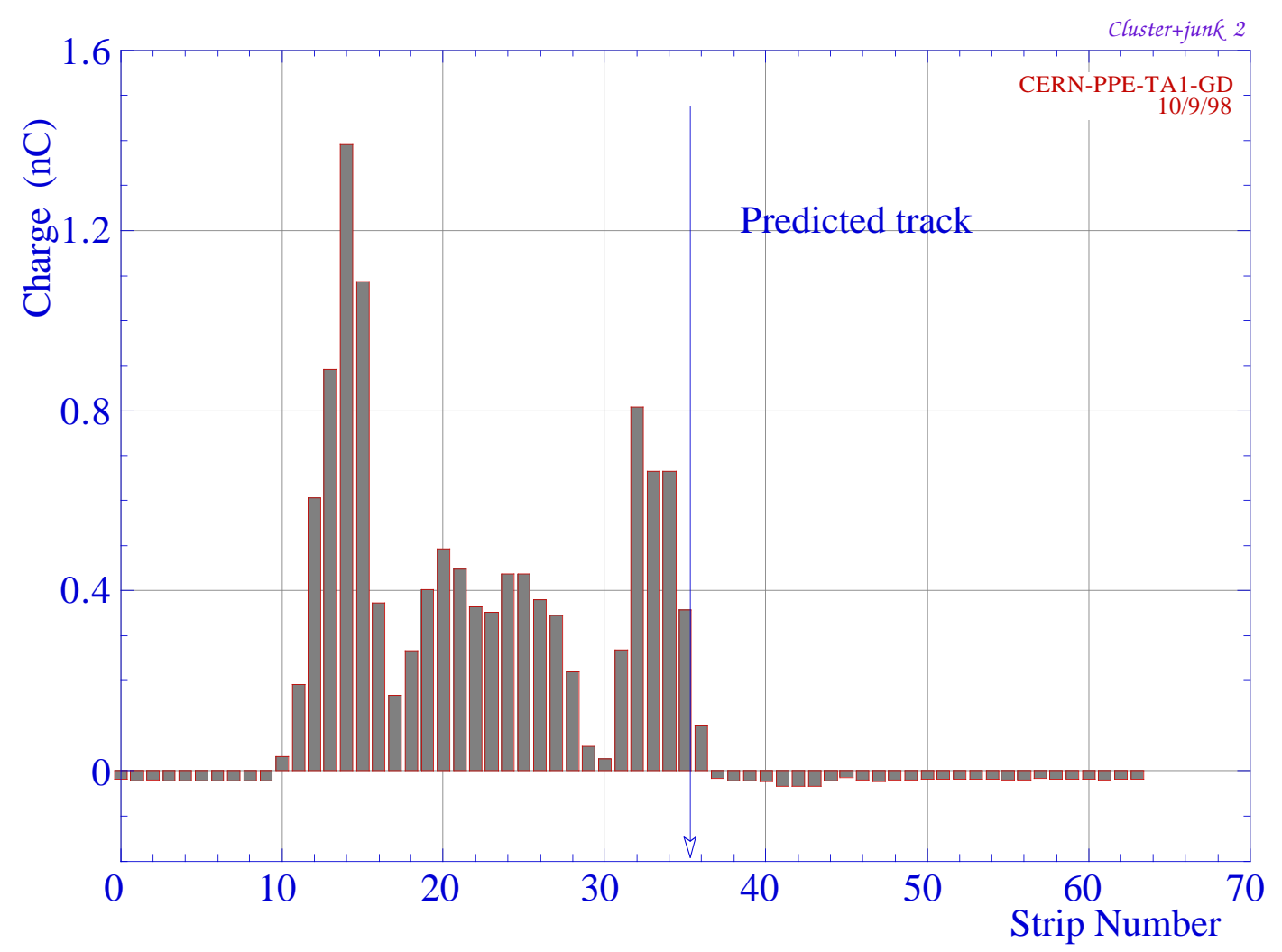


Fig. 14

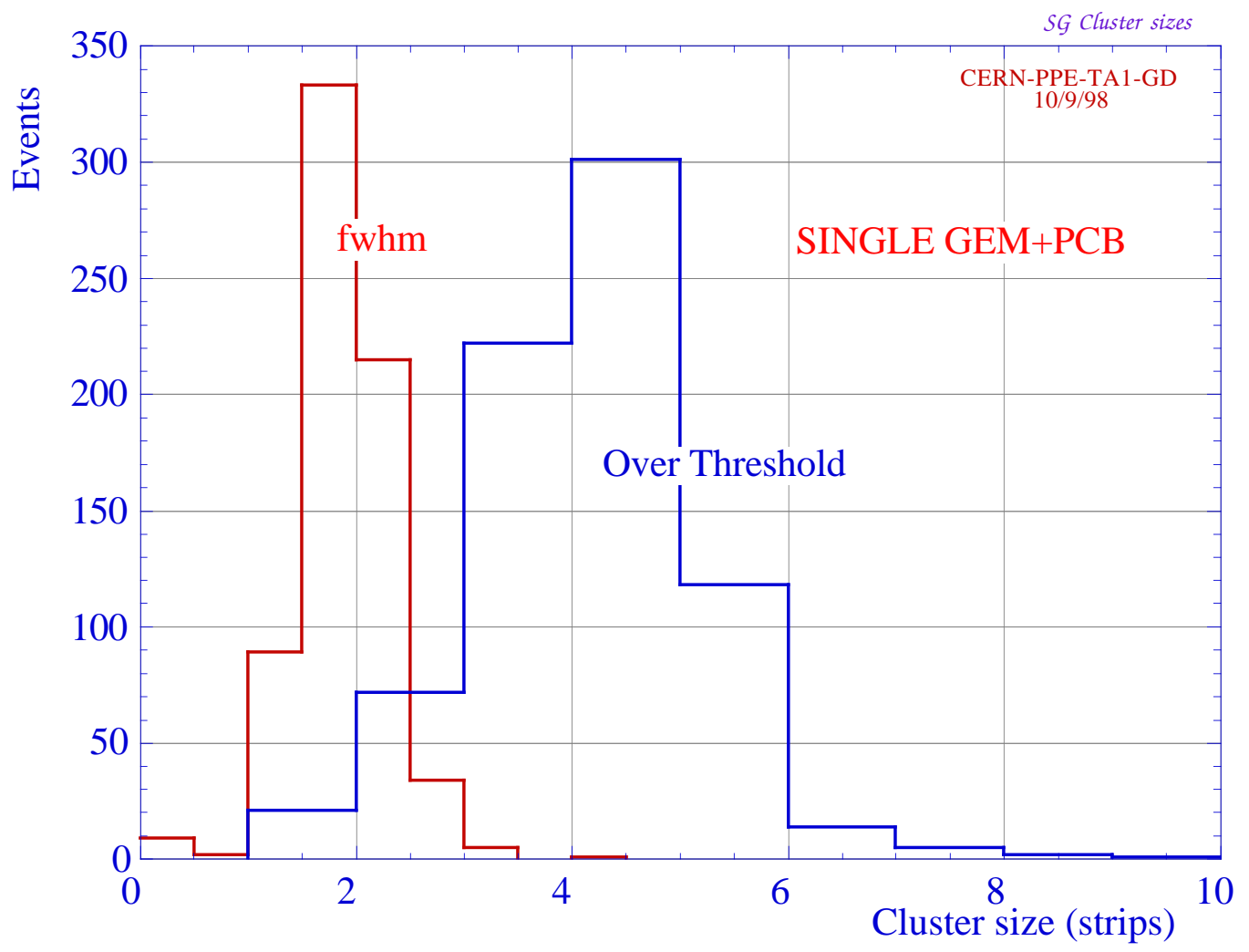

Fig. 15

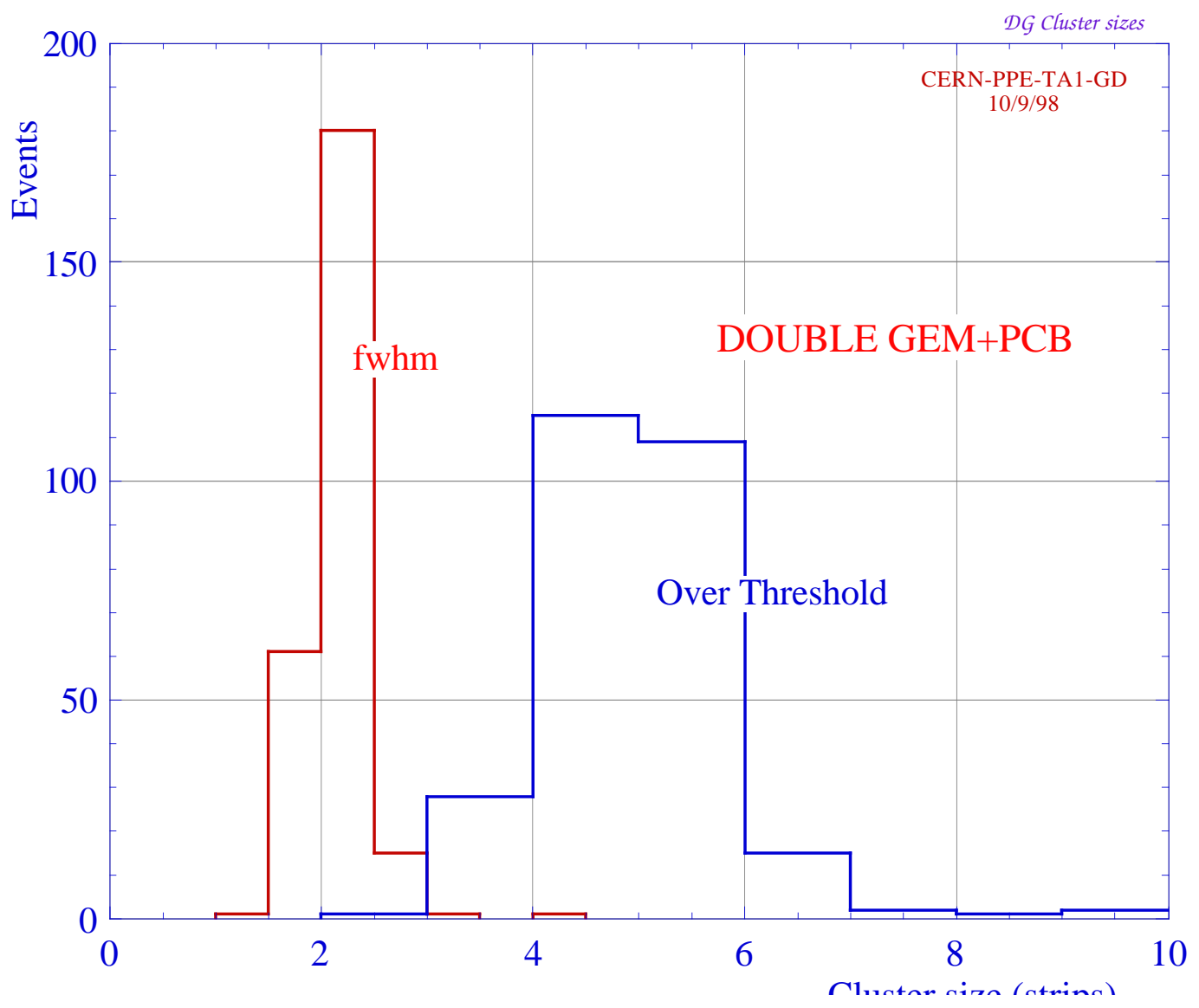


Fig. 16

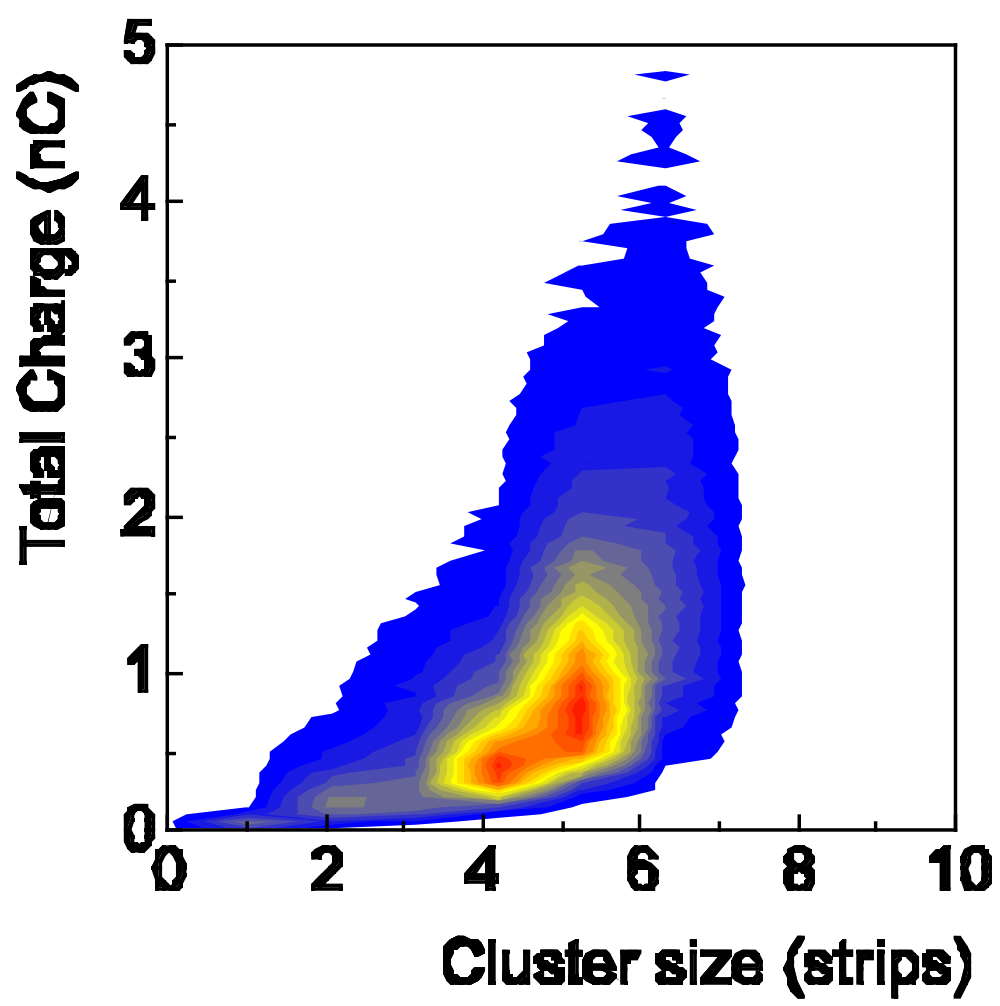

Fig. 17

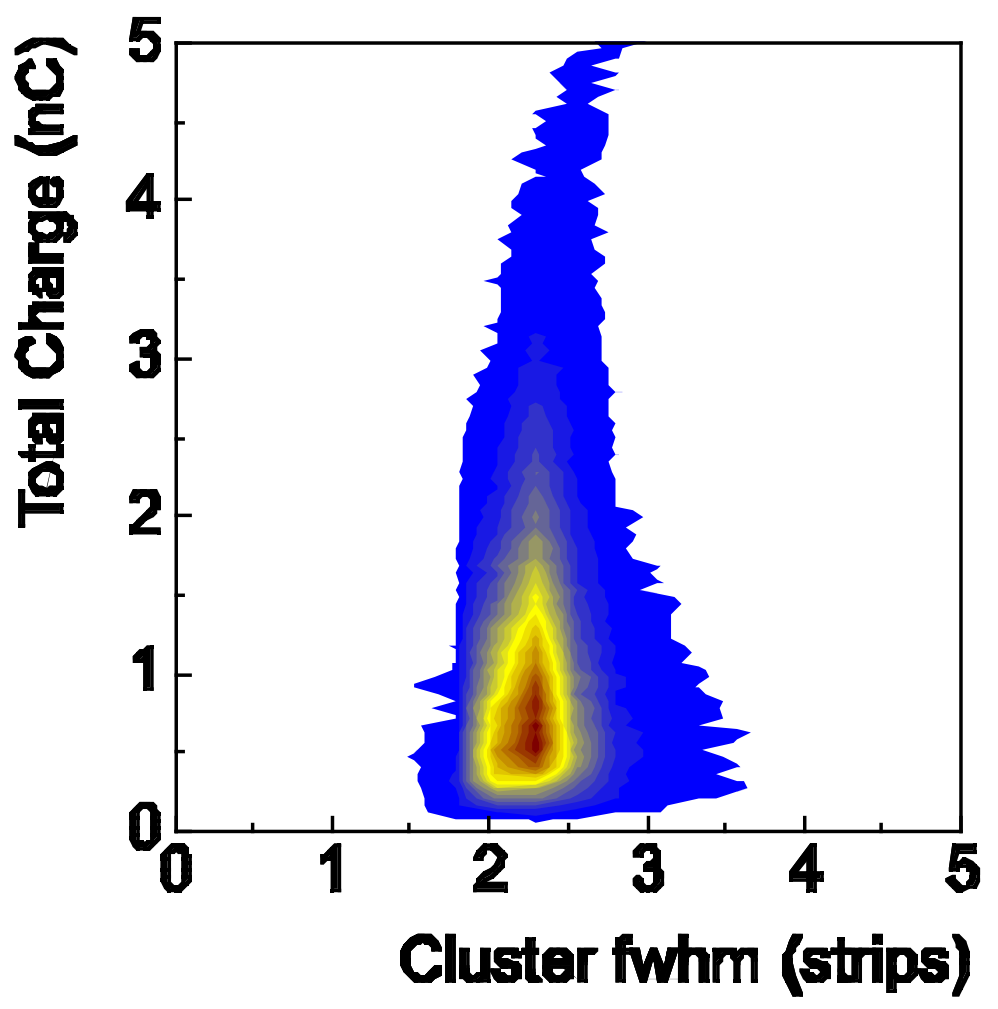




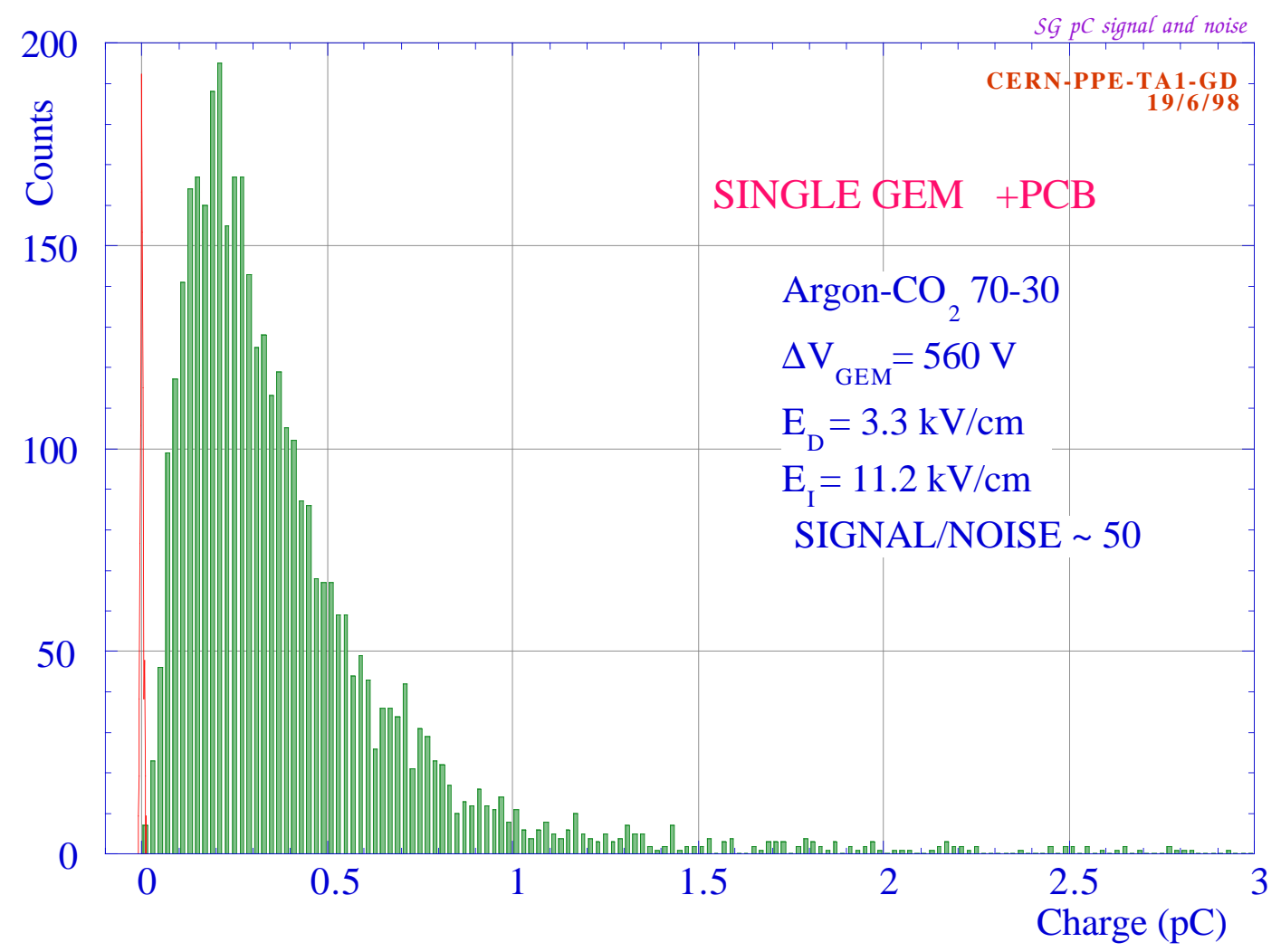

Fig. 19

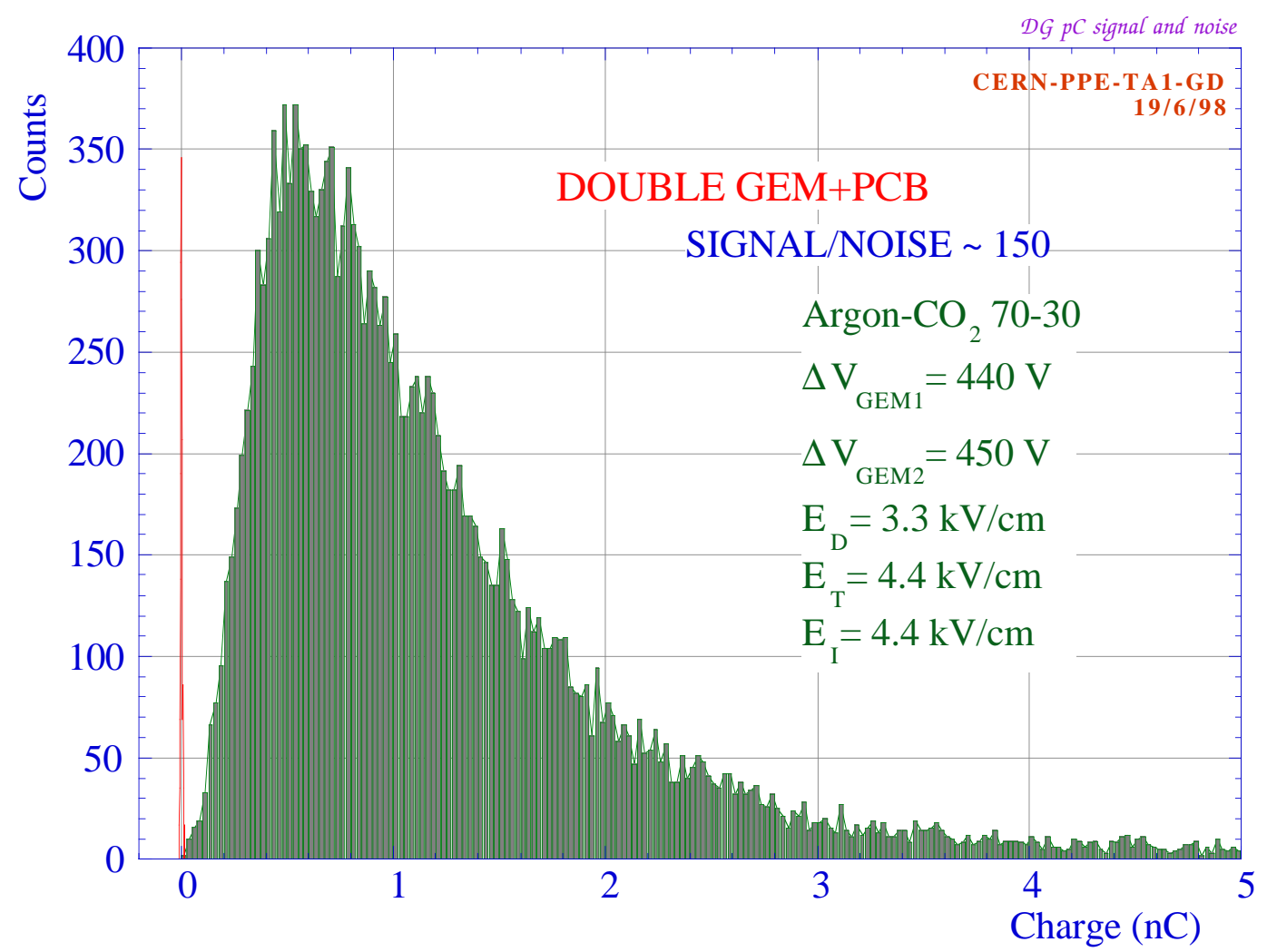

Fig. 20 


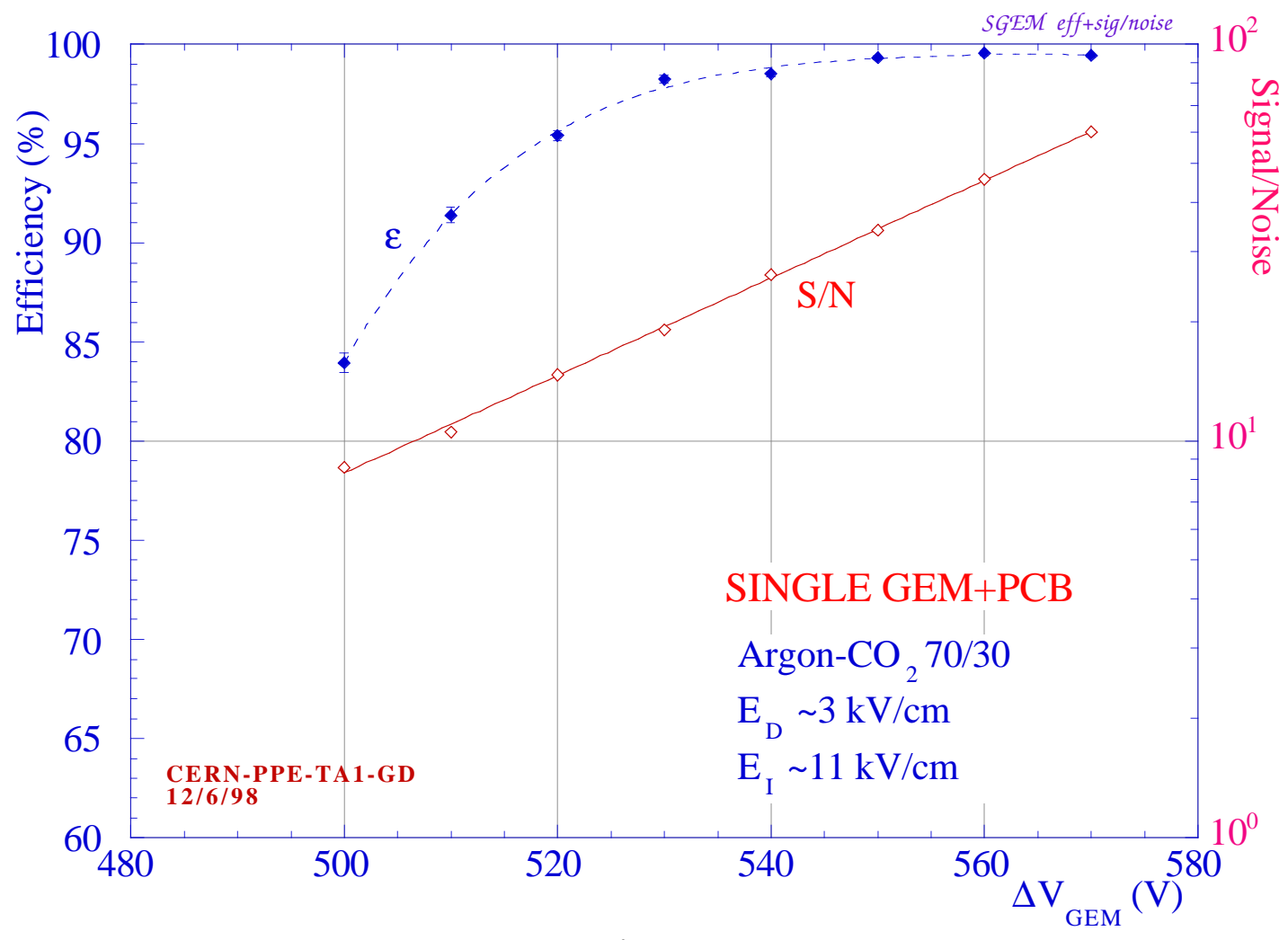

Fig. 21

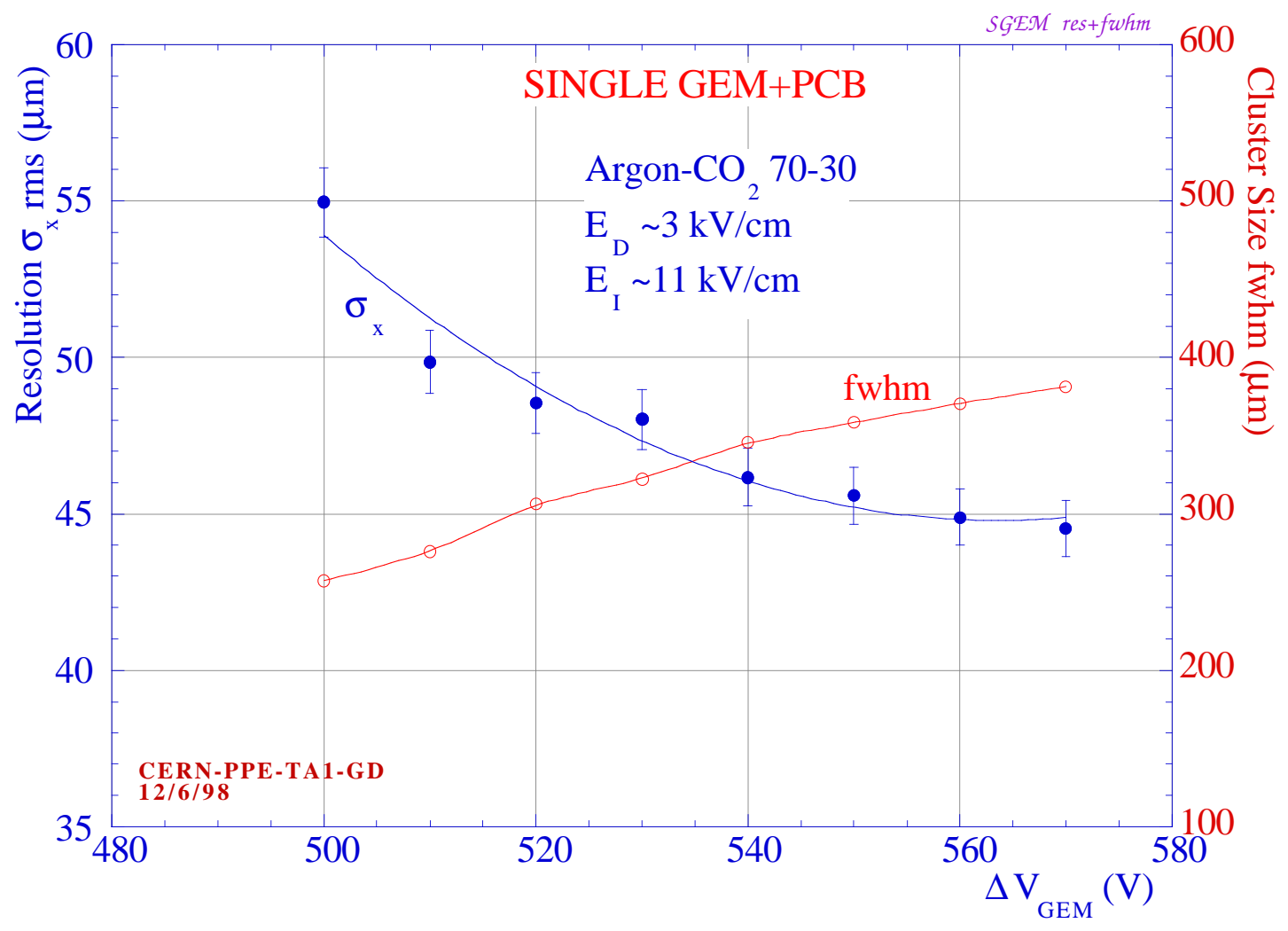

Fig. 22 


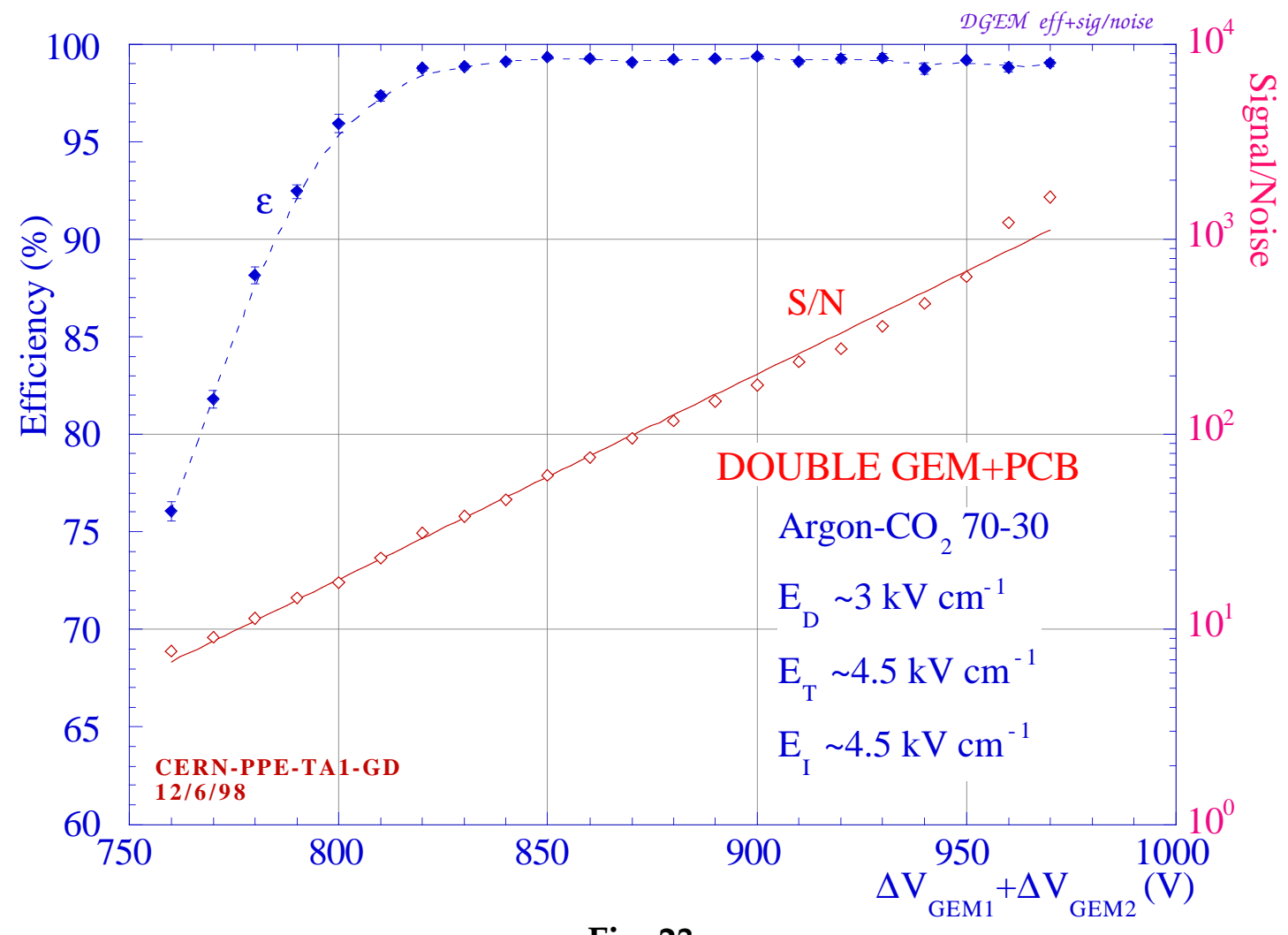

Fig. 23

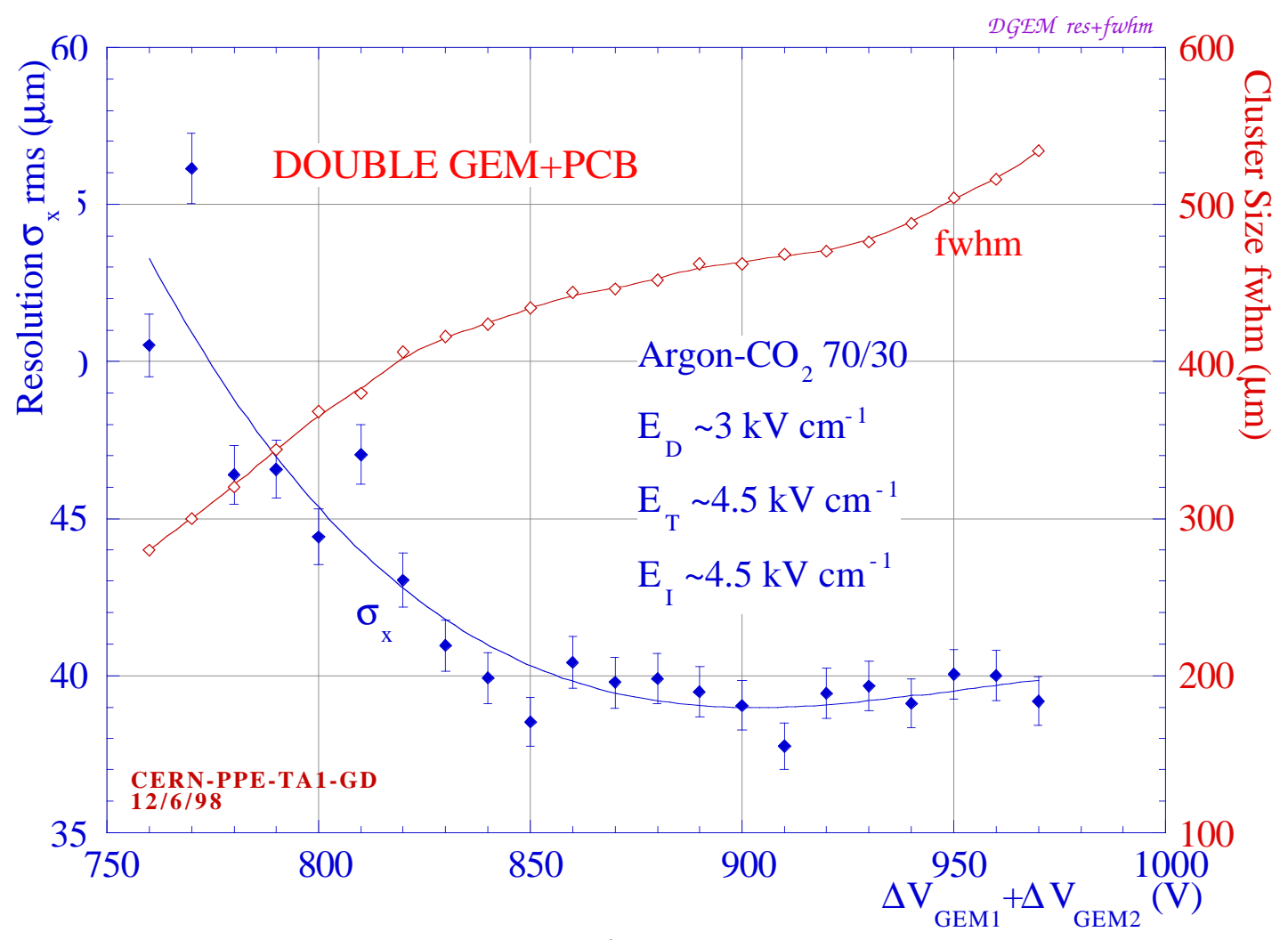

Fig. 24 


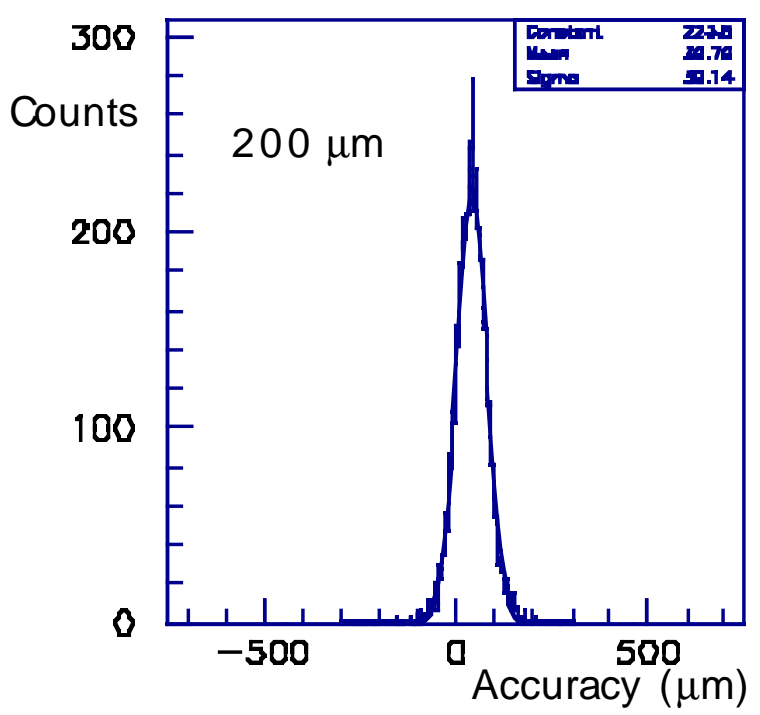

Fig. 25

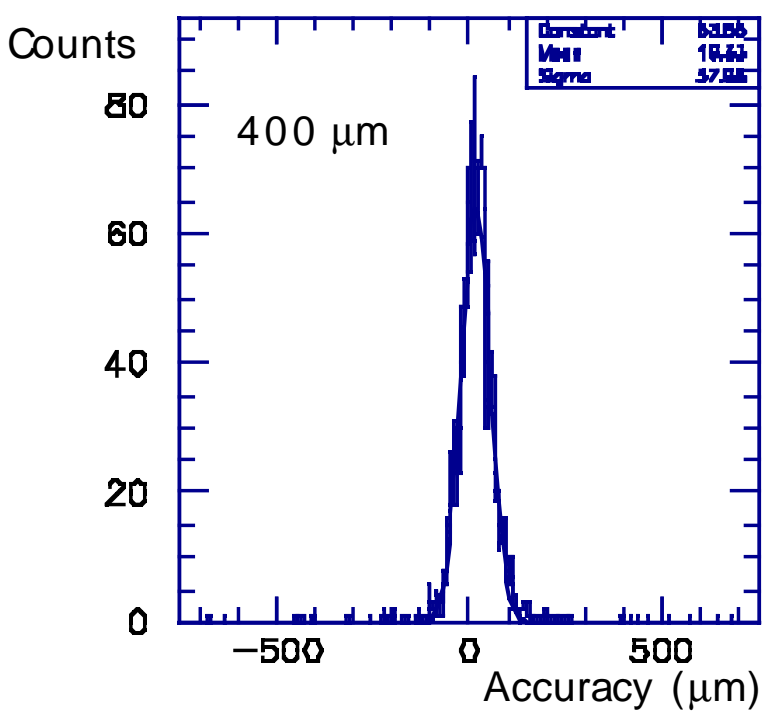

Fig. 26

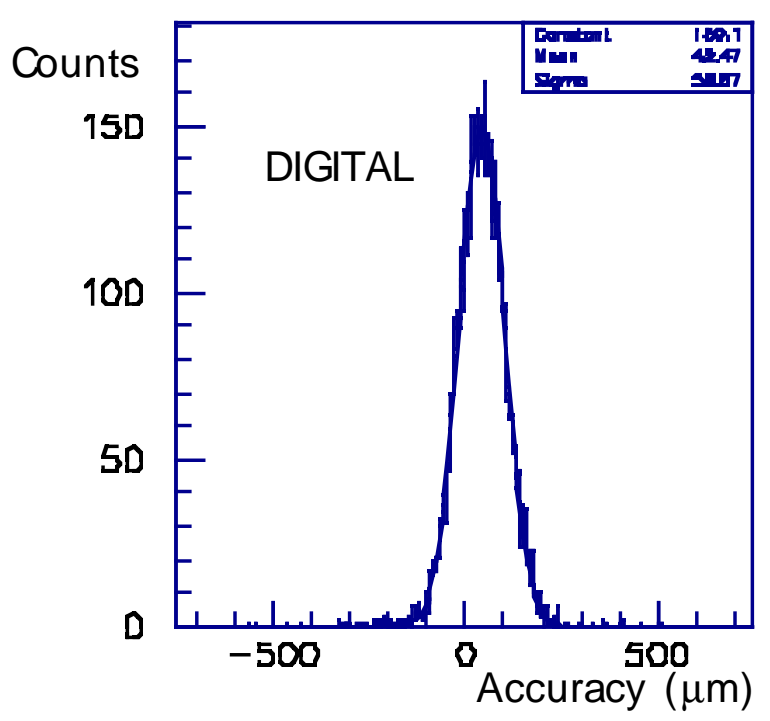

Fig. 27 


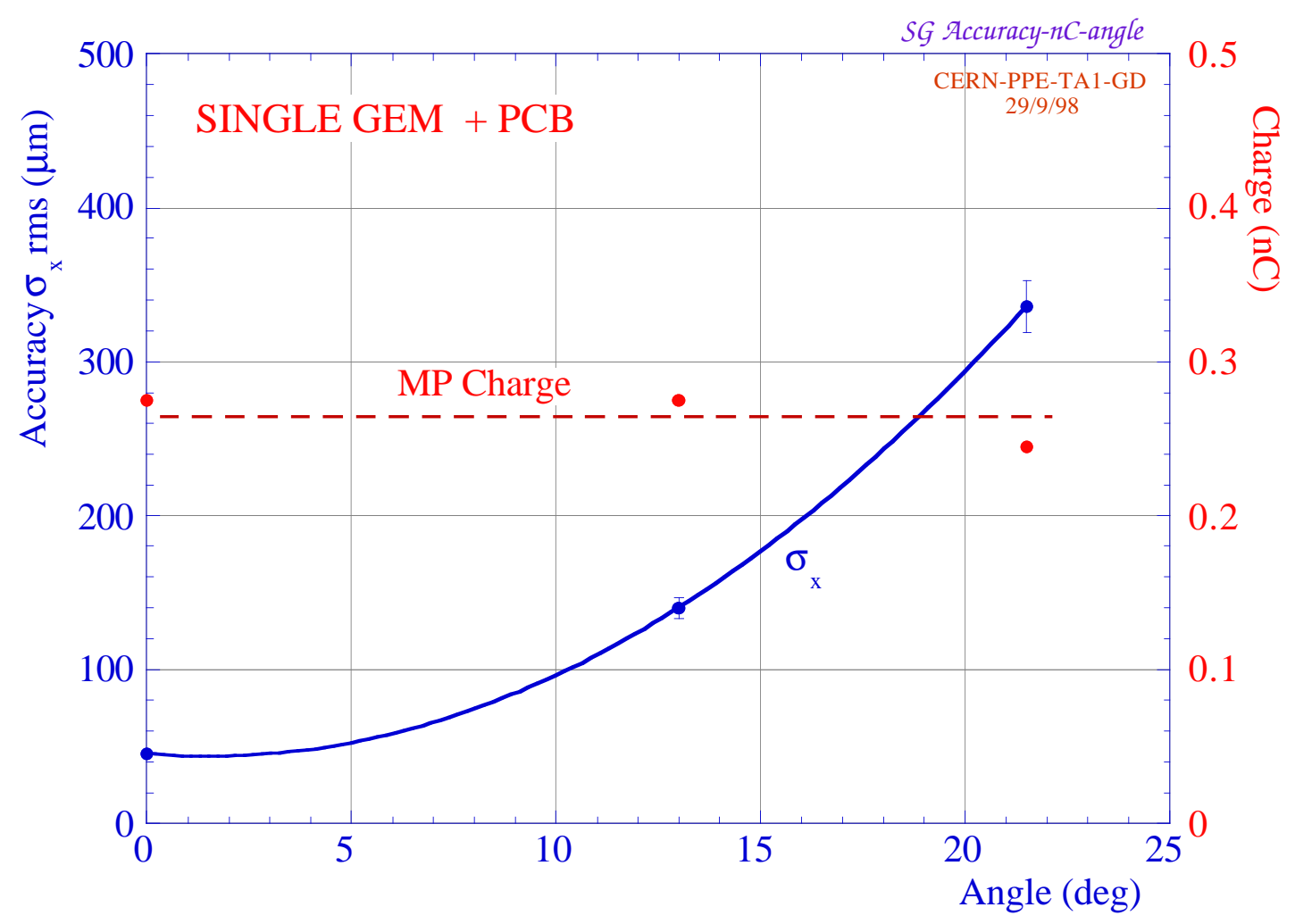

Fig. 28

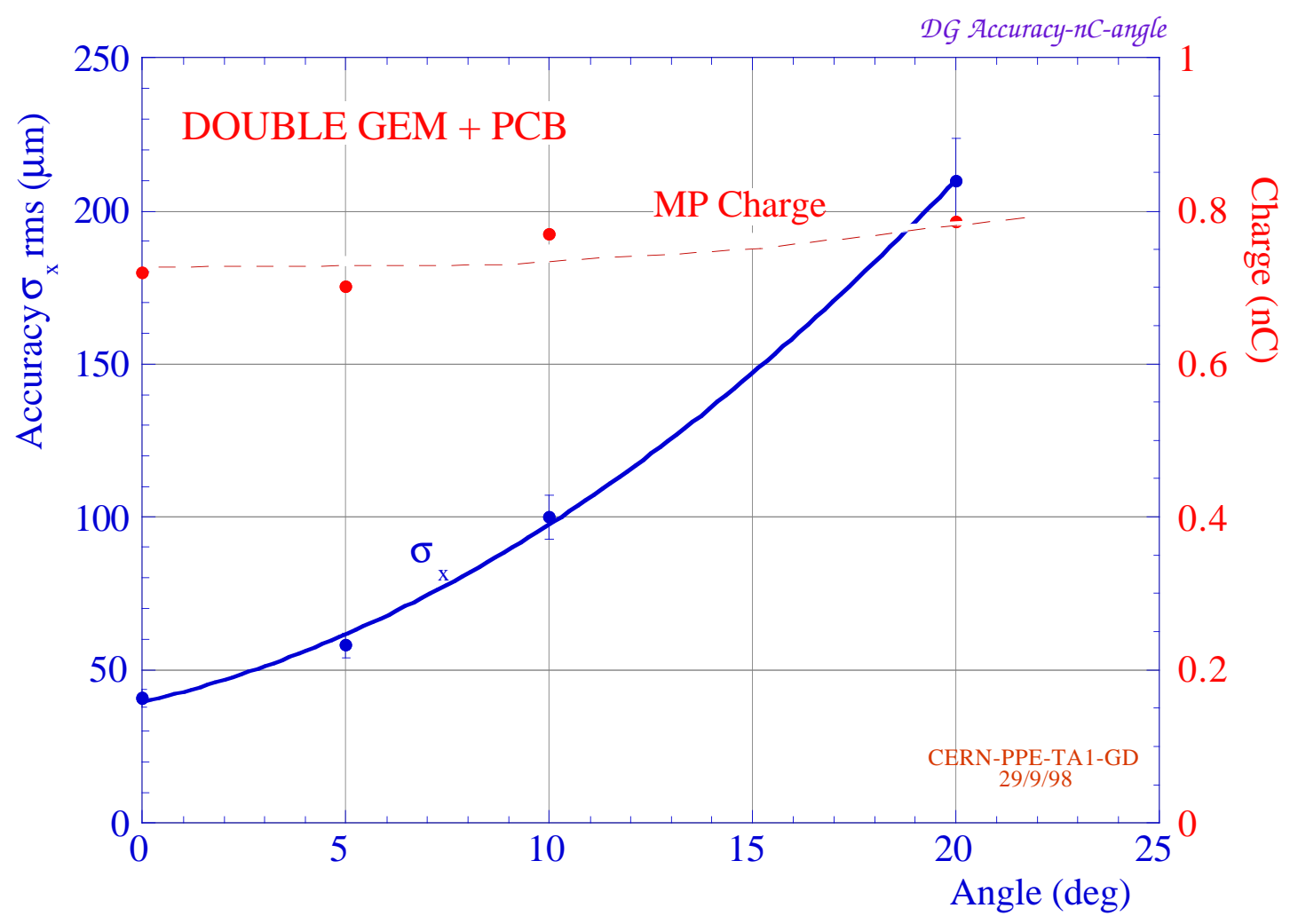

Fig. 29 


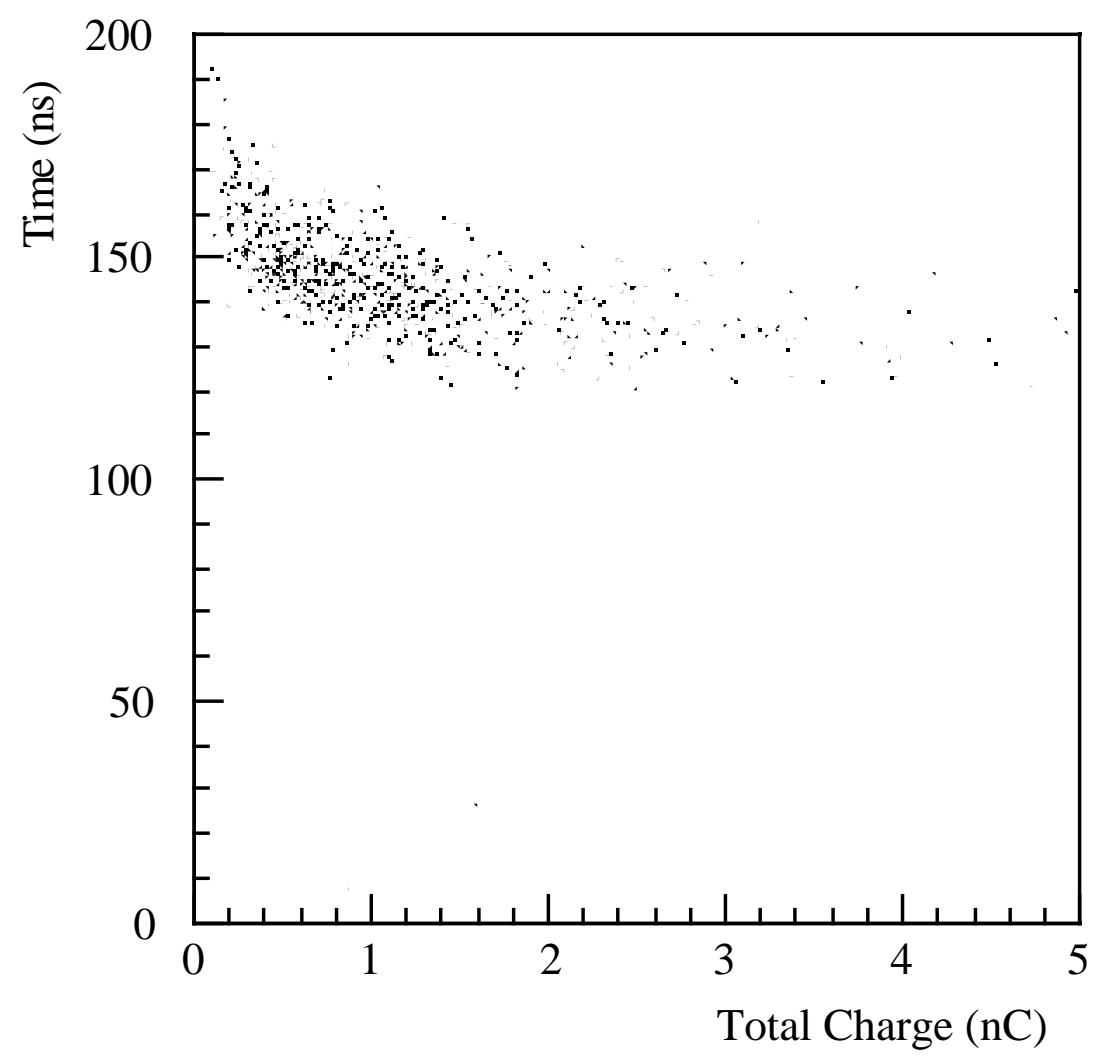

Fig. 30

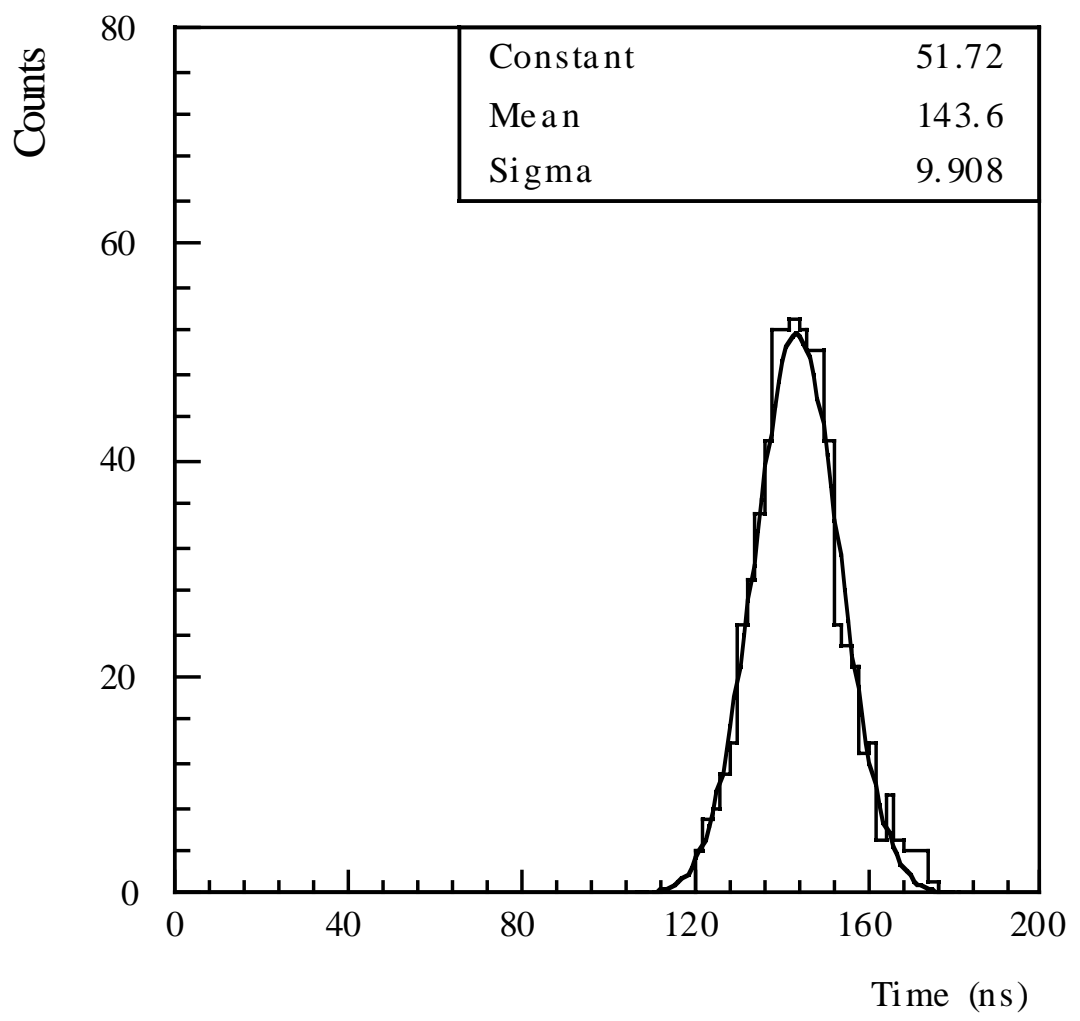

Fig. 31 


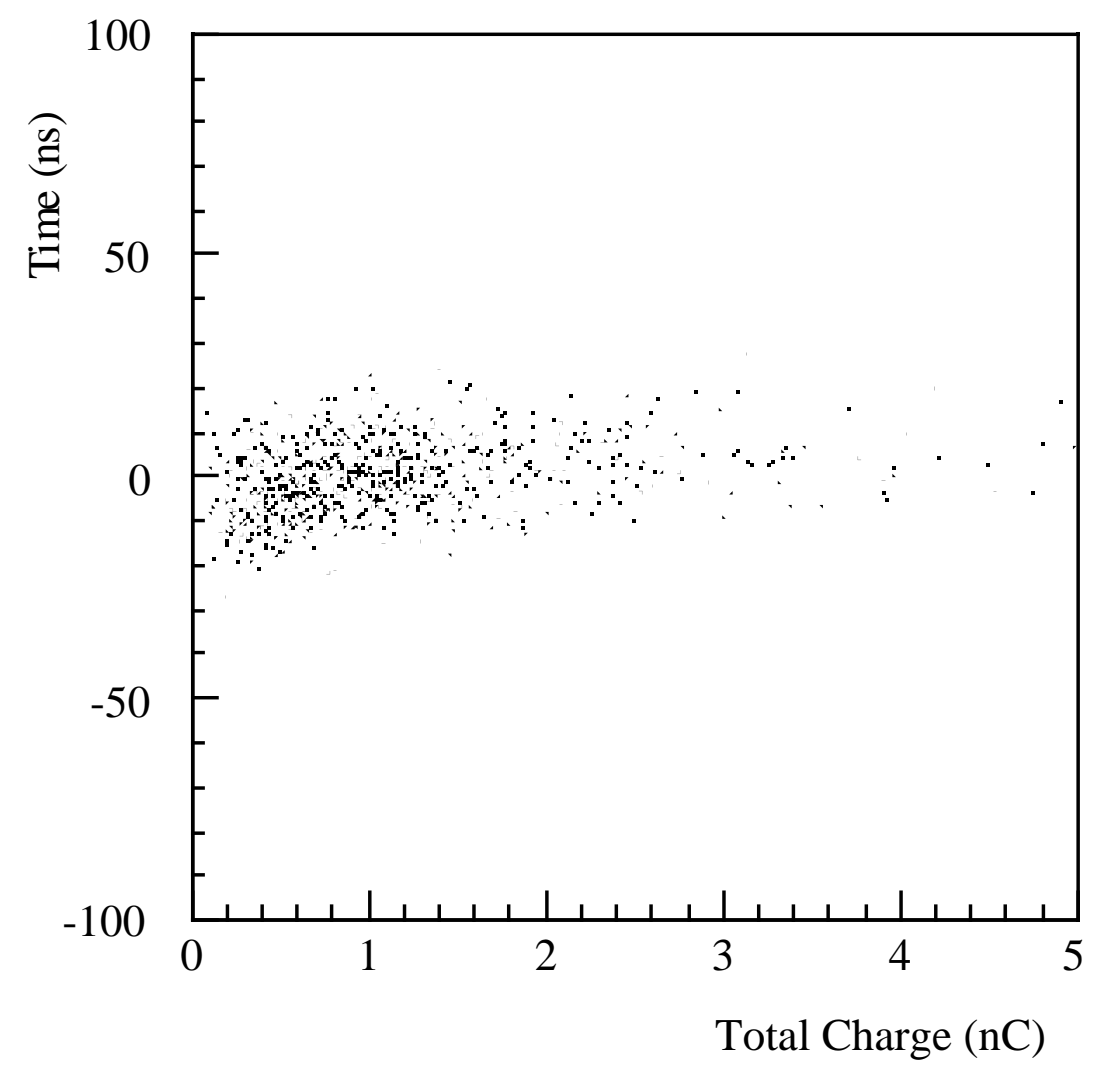

Fig. 32

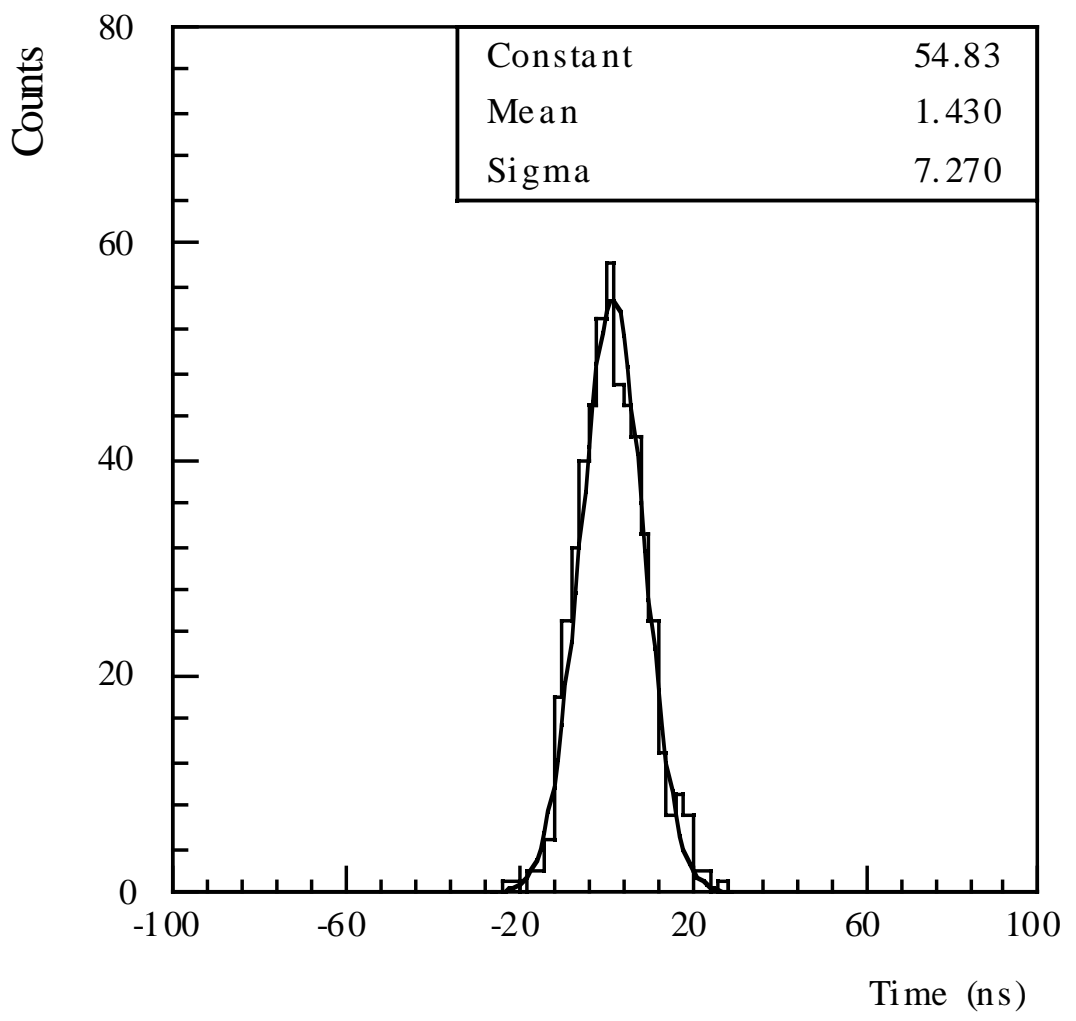

Fig. 33 